\title{
The first law of heterotic stringy black hole mechanics at zeroth order in $\alpha^{\prime}$
}

\author{
Zachary Elgood, Dimitrios Mitsios, Tomás Ortín and David Pereñíguez \\ Instituto de Física Teórica UAM/CSIC, \\ C/ Nicolás Cabrera, 13-15, C.U. Cantoblanco, E-28049 Madrid, Spain \\ E-mail: zachary.elgood@uam.es, di.mitsios@gmail.com, \\ tomas.ortin@csic.es, david.perenniguez@uam.es
}

ABSTRACT: We prove the first law of black hole mechanics in the context of the Heterotic Superstring effective action compactified on a torus to leading order in $\alpha^{\prime}$, using Wald's formalism, covariant Lie derivatives and momentum maps. The Kalb-Ramond field strength of this theory has Abelian Chern-Simons terms which induce Nicolai-Townsend transformations of the Kalb-Ramond field. We show how to deal with all these gauge symmetries deriving the first law in terms of manifestly gauge-invariant quantities. In presence of Chern-Simons terms, several definitions of the conserved charges exist, but the formalism picks up only one of them to play a role in the first law. We study explicitly a non-extremal, charged, black ring solution of pure $\mathcal{N}=1, d=5$ supergravity embedded in the Heterotic Superstring effective field theory.

This work is a first step towards the derivation of the first law at first order in $\alpha^{\prime}$ where, more complicated, non-Abelian, Lorentz ("gravitational") and Yang-Mills ChernSimons terms are included in the Kalb-Ramond field strength. The derivation of a first law is a necessary step towards the derivation of a manifestly gauge-invariant entropy formula which is still lacking in the literature. In its turn, this entropy formula is needed to compare unambiguously macroscopic and microscopic black hole entropies.

Keywords: Black Holes in String Theory, Classical Theories of Gravity, Differential and Algebraic Geometry

ArXiv EPrint: 2012.13323 


\section{Contents}

1 Introduction 1

2 The Heterotic Superstring effective action on $\mathrm{T}^{n}$ at zeroth order in $\alpha^{\prime} \quad 4$

3 Variations of the fields $\quad 6$

$\begin{array}{ll}3.1 \text { Gauge transformations } & 7\end{array}$

3.2 Gauge charges 8

$\begin{array}{lll}3.3 & \text { Diffeomorphisms and covariant Lie derivatives } & 10\end{array}$

$\begin{array}{lll}3.4 & \text { The Wald-Noether charge } & 14\end{array}$

4 Zeroth laws $\quad 16$

$\begin{array}{lll}5 & \text { The first law } & 17\end{array}$

6 Momentum maps for black rings in $d=5 \quad 19$

7 Discussion $\quad 26$

A A truncation of the $d=5$ theory to a $\mathcal{N}=1, d=5$ supergravity $\quad 27$

A.1 Further truncation to pure $\mathcal{N}=1, d=5$ supergravity 31

\section{Introduction}

In ref. [1], Wald showed that, in a theory of gravity invariant under diffeomorphisms, the black hole entropy is essentially the Noether charge associated to that invariance. The proof consists in showing that this charge plays the role of entropy in the first law of black hole mechanics [2]. In presence of matter, though, some terms in the total Noether charge are identified with other terms in the first law and only the "gravitational" part of the Noether charge can be identified with the entropy and, in principle, it is necessary to go through the proof of the first law in order to identify the entropy. In ref. [3], Iyer and Wald studied theories of gravity coupled to matter and found a prescription (henceforth called the Iyer-Wald prescription) to compute directly the entropy. In the derivation of the Iyer-Wald prescription though, it was assumed that all the fields of the theory are tensors, a condition which, in the Standard Model for instance, would only be satisfied by the metric, since the rest of the fields have some kind of gauge freedom, including the Higgs "scalar". If we decide to describe the gravitational field through the Vielbein (as the presence of fermions in the Standard Model demands), not even the gravitational field would be a tensor. 
This problem was first noticed by Jacobson and Mohd [4] in the context of the theory of General Relativity described by a Vielbein. ${ }^{1}$ They solved the problem by "improving" the standard Lie derivative (in the language of $[7,8]$ ) by adding a local Lorentz transformation that covariantizes it. This Lorentz-covariant Lie derivative, also known as Lie-Lorentz derivative occurs naturally in supergravity and it was described in that context for arbitrary Lorentz tensors in ref. $[9]^{2}$ building upon earlier work on the Lie derivative of Lorentz spinors by Lichnerowicz, Kosmann and others [12-18]. A recent application to supergravity, including the fermion fields can be found in ref. [19].

A more general and mathematically rigorous treatment based on the theory of principal bundles was given in ref. [20] by Prabhu, who was motivated by the problems found by Gao in ref. [21]. However, String and Supergravity theories have $p$-form fields with gauge freedom that cannot be described in that framework. Furthermore, the effective action and the field strengths often contain Chern-Simons terms which make the action invariant only up to total derivatives and complicate the gauge transformations of the $p$-form fields. When the Chern-Simons terms depend on the spin (Lorentz) connection, gauge invariance and diffeomorphism invariance become entangled in a very complex form.

One of the simplest theories with a Chern-Simons term in the action is "minimal" $(\mathcal{N}=1) 5$-dimensional supergravity [22], which only contains a 1 -form coupled to gravity. In order to deal with the lack of exact gauge invariance one has to take into account the total derivative in the definition of the Noether current [5]. However, the entropy obtained by this method in ref. [23] in the case of the "gravitational" Chern-Simons terms (both in the action or in the Kalb-Ramond field strength) of the Heterotic Superstring effective action turned out to be gauge-dependent. ${ }^{3}$ This problem was dealt with in ref. [26], albeit in a rather complicated form.

In a recent paper [27] we studied the use of gauge-covariant Lie derivatives in the context of the Einstein-Maxwell theory using momentum maps to construct the derivatives. Momentum maps arise naturally wherever symmetries of a base manifold have to be related to gauge transformations $[10,28]$ and they are unsurprisingly ubiquitous in gauged supergravity. As a matter of fact, the Lie-Lorentz derivative can be constructed in terms of a Lorentz momentum map and in [27] we also used a Maxwell momentum map to construct a Lie-Maxwell derivative, covariant under the gauge transformations of the Maxwell field.

This procedure guarantees the gauge-invariance of the results and, as a byproduct, we found a very interesting relation between momentum maps and generalized zeroth laws also observed, in a completely different language by Prabhu in ref. [20].

In this paper we extend this method to a theory with Abelian Chern-Simons terms in a field strength: the effective action of the Heterotic Superstring compactified on a torus to zeroth order in $\alpha^{\prime}$. This theory can be seen as a generalization of the theory considered by Compère in ref. [6] and as a first step towards dealing with the effective action of the Heterotic Superstring to first order in $\alpha^{\prime}$, which contains non-Abelian and Lorentz ("gravitational") Chern-Simons terms of the kind considered by Tachikawa [29, 30]. The

\footnotetext{
${ }^{1}$ Fields with gauge freedom had already been correctly dealt with in refs. [5, 6], for instance.

${ }^{2}$ See also ref. [10] and, for a more mathematically rigorous point of view, ref. [11].

${ }^{3}$ The same happens when one naively uses the Iyer-Wald prescription, as noticed in [24, 25].
} 
introduction of momentum maps will allow us to obtain invariant results in a rather simple form, basically because they allow us to determine explicitly the gauge parameters that leave invariant all the fields of a given solution [31]. They also allow us to construct forms which are closed on the bifurcation sphere, from which the definitions of the potentials that appear in the first law will follow [6,32]. The closedness of those forms, therefore, plays the role of the generalized zeroth law, albeit restricted to the bifurcation sphere. Hence, we will refer to these properties as the restricted generalized zeroth laws.

As we are going to see in the proof of the first law, there is a very precise, almost clockwork, relation between the closed forms that satisfy the restricted generalized zeroth laws and the definitions of the conserved charges [31,33-35]. Only when both have been correctly identified is it possible to find the first law and identify the entropy.

In theories with Chern-Simons terms, several different definitions of charges have been proposed and used in the literature (see, for instance, ref. [36] and references therein). The proof of the first law demands that we use the so-called Page charge, which in this context is conserved, localized and on-shell gauge invariant. Only when we use this charge definition for the 1-forms, the closed 1-form associated to the KR potentials $\Phi^{i}$ over the bifurcation sphere appears $[6,32]$ and the term $\Phi^{i} \delta \mathcal{Q}_{i}$ of the first law associated to the "dipole charges" [6, 32, 37-40] can be identified.

In theories with "gravitational" Chern-Simons terms, such as the effective action of the Heterotic Superstring at first order in $\alpha^{\prime}$ the same mechanism should play a role in the proof of the first law, but the terms that modify the gravitational charges will contribute to the entropy instead [41]. It is in this precise sense that this work is a first step towards the proof of the first law and the determination of a gauge-invariant entropy formula for that theory. The previous discussion should have made clear that such a formula is not yet available, as we have also explained in refs. [24, 25]. Even though the calculations of some black-hole entropies using the Iyer-Wald prescription seem to give the right value of the entropy in some cases, ${ }^{4}$ it is clear that the results obtained using an entropy formula which is not gauge-invariant cannot be trusted in general. It is also clear that the comparison between entropies computed through macroscopic and microscopic methods [44] only make sense if both computations are reliable, and furthermore, only if the relation between the parameters of the black hole solution and of the microscopic theory is well understood. At first order in $\alpha^{\prime}$, there is no full-proof entropy formula, as we have explained, and the identification of the parameters of the black-hole solutions (charges) with the numbers of branes and other parameters that appear in the microscopic entropy, has issues that still have not been fully understood [45]. This is one of the main motivations for this work.

\footnotetext{
${ }^{4}$ In ref. [42] it was shown that the entropy of the $\alpha^{\prime}$-corrected non-extremal Reissner-Nordström black hole based in the string embedding of ref. [43], computed with the entropy formula derived in ref. [24] using the Iyer-Wald prescription satisfies the thermodynamic relation $\partial S / \partial M=T^{-1}$. That entropy formula is not invariant under Lorentz transformations, though. In a general frame it will give wrong values for the entropy and the reason why it gives the right value in that particular case, in the particular frame in which the calculation was carried out, sill needs to be explained [41]. The same entropy formula has been used to compute the entropy of some $\alpha^{\prime}$-corrected extremal black holes and the results, although reasonable, cannot be tested using the same relation.
} 
This paper is organized as follows: in section 2 we introduce the effective action of the Heterotic Superstring compactified on a torus at leading order in $\alpha^{\prime}$. In section 3 we study the action of the symmetries of the theory on the fields, the parameters of the transformations that leave all of them invariant, and compute the associated conserved charges, including the Wald-Noether charge. In section 4 we study the restricted generalized zeroth laws that we will use in the proof of the first law in section 5. In section 6 we consider as an example the charged, non-extremal, 5 -dimensional black ring solution of pure $\mathcal{N}=1, d=5$ supergravity of ref. [46] and compute its momentum maps. Section 7 contains a brief discussion of our results. In the appendix we show how the Heterotic Superstring effective action compactified on $\mathrm{T}^{4} \times \mathrm{S}^{1}$ (trivial compactification on $\mathrm{T}^{4}$ ) can be understood as a model $\mathcal{N}=1, d=5$ supergravity coupled to two vector supermultiplets, which provides an embedding of this model into the Heterotic Superstring effective action. We also show how this model can be consistently trunctated to pure $\mathcal{N}=1, d=5$ supergravity. Again, this provides an embedding of pure $\mathcal{N}=1, d=5$ supergravity and, in particular of the black ring solution of ref. [46] into the Heterotic Superstring effective action, so we can apply the formulae and results obtained in the main body of the paper to that solution.

\section{The Heterotic Superstring effective action on $\mathrm{T}^{n}$ at zeroth order in $\alpha^{\prime}$}

When the effective action of the Heterotic Superstring at leading order in $\alpha^{\prime}$ is compactified on a $\mathrm{T}^{n}$, it describes the dynamics of the $(10-n)$-dimensional (string-frame) metric $g_{\mu \nu}$, Kalb-Ramond 2-form $B_{\mu \nu}$, dilaton field $\phi$, Kaluza-Klein (KK) and winding 1-forms $A^{m}{ }_{\mu}$ and $B_{m \mu}$, respectively, and the scalars that parametrize the $\mathrm{O}(n, n) / \mathrm{O}(n) \times \mathrm{O}(n)$ coset space, collected in the symmetric $\mathrm{O}(n, n)$ matrix $M$ that we will write with upper $\mathrm{O}(n, n)$ indices $I, J, \ldots$ as $M^{I J}$. This means that $M$ satisfies

$$
M^{I J} \Omega_{J K} M^{K L} \Omega_{L M}=\delta^{I}{ }_{M},
$$

where

$$
\left(\Omega_{I J}\right) \equiv\left(\begin{array}{cc}
0 & \mathbb{1}_{n \times n} \\
\mathbb{1}_{n \times n} & 0
\end{array}\right),
$$

is the off-diagonal form of the $\mathrm{O}(n, n)$ metric. Eq. (2.1) implies that

$$
M_{I J} \equiv\left(M^{-1}\right)_{I J}=\Omega_{I K} M^{K L} \Omega_{L J} .
$$

Using the notation and conventions of refs. [10, 25] (in particular, for differential forms, we use those of ref. [27]), and calling the physical scalars in $M_{I J} \phi^{x}$, the action of the $d=(10-n)$-dimensional takes the form

$$
\begin{aligned}
S\left[e^{a}, B, \phi, \mathcal{A}^{I}, \phi^{x}\right]= & \frac{g_{s}^{(d) 2}}{16 \pi G_{N}^{(d)}} \int e^{-2 \phi}\left[(-1)^{d-1} \star\left(e^{a} \wedge e^{b}\right) \wedge R_{a b}-4 d \phi \wedge \star d \phi\right. \\
& \left.-\frac{1}{8} d M_{I J} \wedge \star d M^{I J}+(-1)^{d} \frac{1}{2} M_{I J} \mathcal{F}^{I} \wedge \star \mathcal{F}^{J}+\frac{1}{2} H \wedge \star H\right] \\
\equiv & \int \mathbf{L} .
\end{aligned}
$$


In this action $e^{a}=e^{a}{ }_{\mu} d x^{\mu}$ are the string-frame Vielbeins, $\star$ stands for the Hodge dual and, therefore

$$
\star\left(e^{a} \wedge e^{b}\right)=\frac{1}{(d-2) !} \epsilon_{c_{1} \cdots c_{d-2}}^{a b} e^{c_{1}} \wedge \cdots \wedge e^{c_{d-2}} .
$$

Furthermore, $\omega^{a b}=\omega_{\mu}^{a b} d x^{\mu}$ is the Levi-Civita spin connection ${ }^{5}$ and $R^{a b}=\frac{1}{2} R_{\mu \nu}{ }^{a b} d x^{\mu} \wedge d x^{\nu}$ is its field strength (the curvature) 2-form, defined as

$$
R^{a b} \equiv d \omega^{a b}-\omega^{a}{ }_{c} \wedge \omega^{c b} .
$$

$g_{s}^{(d)}$ and $G_{N}^{(d)}$ are, respectively, the $d=(10-n)$-dimensional string coupling and Newton constant. ${ }^{6} \mathcal{F}^{I}$ is the $\mathrm{O}(n, n)$ vector of the 2-form field strengths of the KK and winding vectors

$$
\mathcal{F}^{I} \equiv\left(\begin{array}{c}
F^{m} \\
G_{m}
\end{array}\right), \quad F^{m}=d A^{m}, \quad G_{m}=d B_{m},
$$

which can also be defined in terms of the $\mathrm{O}(n, n)$ vector of 1 -forms denoted by $\mathcal{A}^{I}$

$$
\mathcal{A}^{I} \equiv\left(\begin{array}{c}
A^{m} \\
B_{m}
\end{array}\right), \quad \mathcal{F}^{I}=d \mathcal{A}^{I} .
$$

$H$ is the Kalb-Ramond 3-form field strength, defined by

$$
H \equiv d B-\frac{1}{2} \mathcal{A}_{I} \wedge d \mathcal{A}^{I}, \quad \mathcal{A}_{I}=\Omega_{I J} \mathcal{A}^{J}
$$

The kinetic term of the scalars $\phi^{x}$ that parametrize the $\mathrm{O}(n, n) /(\mathrm{O}(n) \times \mathrm{O}(n))$ coset space can also be written in the form

$$
-\frac{1}{8} d M_{I J} \wedge \star d M^{I J}=\frac{1}{2} g_{x y} d \phi^{x} \wedge \star d \phi^{y}
$$

where the metric $g_{x y}(\phi)$ is given by

$$
g_{x y} \equiv \frac{1}{4}\left(\partial_{x} M_{I K} M^{K J}\right)\left(\partial_{y} M_{J K} M^{K I}\right) .
$$

Under a general variation of the fields, the action varies as

$$
\delta S=\int\left\{\mathbf{E}_{a} \wedge \delta e^{a}+\mathbf{E}_{B} \wedge \delta B+\mathbf{E}_{\phi} \delta \phi+\mathbf{E}_{I} \wedge \delta \mathcal{A}^{I}+\mathbf{E}_{x} \delta \phi^{x}+d \mathbf{\Theta}(\varphi, \delta \varphi)\right\}
$$

\footnotetext{
${ }^{5}$ It is antisymmetric $\omega^{a b}=-\omega^{b a}$ and satisfies $D e^{a}=d e^{a}-\omega^{a}{ }_{b} \wedge e^{b}=0$. We are using the second-order formalism.

${ }^{6}$ They are related to the 10 -dimensional constants through the volume of the $\mathrm{T}^{n}, V_{n}$, by

$$
\begin{aligned}
g_{s}^{2} & =V_{n} /\left(2 \pi \ell_{s}\right)^{n} g_{s}^{(d) 2}, \\
G_{N}^{(10)} & =G_{N}^{(d)} V_{n} .
\end{aligned}
$$


where, suppressing the factors of $g^{(d) 2}\left(16 \pi G_{N}^{(d)}\right)^{-1}$ for simplicity, the Einstein equations $\mathbf{E}_{a}$ are given by

$$
\begin{aligned}
\mathbf{E}_{a}= & e^{-2 \phi} \imath_{a} \star\left(e^{c} \wedge e^{d}\right) \wedge R_{c d}-2 \mathcal{D}\left(\imath_{b} d e^{-2 \phi}\right) \wedge \star\left(e^{b} \wedge e^{c}\right) g_{c a} \\
& +(-1)^{d-1} 4 e^{-2 \phi}\left(\imath_{a} d \phi \star d \phi+d \phi \wedge \imath_{a} \star d \phi\right) \\
& +\frac{(-1)^{d}}{2} e^{-2 \phi} g_{x y}\left(\imath_{a} d \phi^{x} \star d \phi^{y}+d \phi^{x} \wedge \imath_{a} \star d \phi^{y}\right) \\
& +\frac{1}{2} e^{-2 \phi} M_{I J}\left(\imath_{a} \mathcal{F}^{I} \wedge \star \mathcal{F}^{J}-\mathcal{F}^{I} \wedge \imath_{a} \star \mathcal{F}^{J}\right) \\
& +\frac{(-1)^{d}}{2} e^{-2 \phi}\left(\imath_{a} H \wedge \star H+H \wedge \imath_{a} \star H\right),
\end{aligned}
$$

the equations of motion of the matter fields are given by

$$
\begin{aligned}
& \mathbf{E}_{B}=-d\left(e^{-2 \phi} \star H\right), \\
& \mathbf{E}_{\phi}=8 d\left(e^{-2 \phi} \star d \phi\right)-2 \mathbf{L}, \\
& \mathbf{E}_{I}=\tilde{\mathbf{E}}_{I}+\frac{1}{2} \mathbf{E}_{B} \wedge \mathcal{A}_{I}, \\
& \tilde{\mathbf{E}}_{I} \equiv-\left\{d\left(e^{-2 \phi} M_{I J} \star \mathcal{F}^{J}\right)+(-1)^{d-1} e^{-2 \phi} \star H \wedge \mathcal{F}_{I}\right\}, \\
& \mathbf{E}_{x}=-g_{x y}\left[d\left(e^{-2 \phi} \star d \phi^{y}\right)+e^{-2 \phi} \Gamma_{z w^{y}} d \phi^{z} \wedge \star d \phi^{w}\right]+\frac{(-1)^{d}}{2} e^{-2 \phi} \partial_{x} M_{I J} \mathcal{F}^{I} \wedge \star \mathcal{F}^{J},
\end{aligned}
$$

and

$$
\begin{aligned}
\Theta(\varphi, \delta \varphi)= & -e^{-2 \phi} \star\left(e^{a} \wedge e^{b}\right) \wedge \delta \omega_{a b}+2 \imath_{a} d e^{-2 \phi} \star\left(e^{a} \wedge e^{b}\right) \wedge \delta e_{b} \\
& -8 e^{-2 \phi} \star d \phi \delta \phi-\frac{1}{4} e^{-2 \phi} \star d M^{I J} \delta M_{I J} \\
& +e^{-2 \phi} M_{I J} \star \mathcal{F}^{J} \wedge \delta \mathcal{A}^{I}+e^{-2 \phi} \star H \wedge\left(\delta B+\frac{1}{2} \mathcal{A}_{I} \wedge \delta \mathcal{A}^{I}\right) .
\end{aligned}
$$

The equations of motion of the 1-forms $\mathbf{E}_{I}$ can be written in the alternative form

$$
\mathbf{E}_{I}=-d\left\{e^{-2 \phi} M_{I J} \star \mathcal{F}^{J}+\star H \wedge \mathcal{A}_{I}\right\}-\frac{1}{2} \mathbf{E}_{B} \wedge \mathcal{A}_{I} .
$$

This form appears naturally in the definition of the electric charges eq. (3.15).

Here, and in what follows, $\varphi$ stands for all the fields of the theory. $\mathbf{E}_{\varphi}$ denotes collectively all their equations of motion.

\section{Variations of the fields}

In this section we are going to study the transformations of the fields under the different symmetries of the action and determine which parameters of the transformations leave a complete field configuration invariant. The conserved charges of those configurations will be associated to those parameters. As a general rule, only if one combines several transformations can one find parameters that simultaneously leave all the fields invariant. 
The simplest case in which this happens will involve the gauge transformations of the 1-form fields: the parameters that leave them invariant do not leave the KR field invariant at the same time, unless we perform a KR gauge transformation with a parameter related to that of the other gauge symmetry. As a result, there is an additional term in the formula that gives the electric charges, but it is the presence of this additional term that guarantees the conservation of the charge and the independence of the integration surface (as long as we do not include sources, that is, on-shell).

The transformation of several fields under diffeomorphisms must also be supplemented by "compensating" gauge transformations, including local Lorentz transformations if we want all the fields to be left invariant by those generating isometries (Killing vectors). There are several ways of understanding this need but we believe that the most fundamental is to realize that fields with gauge freedoms (i.e. all fields except for the metric and the dilaton field) are not tensors and do not transform as such under diffeomorphisms. The "compensating gauge transformations" can be seen as gauge transformations induced by the diffeomorphisms. Only when they are properly taken into account can one find Killing vector fields that leave all the fields invariant. Furthermore, only then the vanishing of the variations of the fields is invariant under gauge transformations. A more detailed discussion and additional references to this topic can be found in ref. [27]. The conserved charge associated to diffeomorphisms, the Wald-Noether charge, will therefore include terms related to gauge symmetries and their associated conserved charges, which will ultimately contribute to the first law.

As we will see, only when all these details are properly taken into account can the first law be proven and the entropy identified.

We start by describing the gauge symmetries of the theory (other than diffeomorphisms) and the associated conserved charges.

\subsection{Gauge transformations}

The gauge transformations of the fields are

$$
\begin{aligned}
\delta_{\sigma} e^{a} & =\sigma^{a}{ }_{b} e^{b}, \\
\delta_{\chi} \mathcal{A}^{I} & =d \chi^{I}, \\
\delta B & =\left(\delta_{\Lambda}+\delta_{\chi}\right) B=d \Lambda+\frac{1}{2} \chi_{I} d \mathcal{A}^{I},
\end{aligned}
$$

where $\sigma^{(a b)}(x)=0$ are the parameters of local Lorentz transformations, $\chi^{I}(x)$ is a $\mathrm{O}(n, n)$ vector if scalar gauge parameters and $\Lambda=\Lambda_{\mu}(x) d x^{\mu}$ is a 1-form gauge parameter. They leave invariant the field strengths $\mathcal{F}^{I}$ and $H$, but they induce the following transformations on the spin connection and curvature

$$
\begin{aligned}
& \delta_{\sigma} \omega^{a b}=\mathcal{D} \sigma^{a b}=d \sigma^{a b}-2 \omega^{[a \mid}{ }_{c} \sigma^{c \mid b]}, \\
& \delta_{\sigma} R^{a b}=2 \sigma^{[a \mid}{ }_{c} R^{c \mid b]} .
\end{aligned}
$$

For the sake of completeness and later use, we quote the Ricci identity in our conventions:

$$
\mathcal{D D} \sigma^{a b}=-2 R^{[a \mid}{ }_{c} \sigma^{c \mid b]}=\delta_{\sigma} R^{a b} .
$$


The action is manifestly invariant under these gauge transformations. This leads to the following Noether identities

$$
\begin{aligned}
\mathbf{E}^{[a} \wedge e^{b]} & =0, \\
d \tilde{\mathbf{E}}_{I}+(-1)^{d} \mathbf{E}_{B} \wedge \mathcal{F}_{I} & =0, \\
d \mathbf{E}_{B} & =0,
\end{aligned}
$$

\subsection{Gauge charges}

Let us study the conserved charges associated to the gauge transformations $\delta_{\chi}, \delta_{\Lambda}$ and, for the sake of completeness, $\delta_{\sigma}$, starting with $\delta_{\Lambda}$, which is simpler to deal with.

The variation of the action under $\delta_{\Lambda}$ transformations follows from eqs. (2.13) and (2.16)

$$
\begin{aligned}
\delta_{\Lambda} S & =\int\left\{\mathbf{E}_{B} \wedge \delta_{\Lambda} B+d\left(e^{-2 \phi} \star H \wedge \delta_{\Lambda} B\right)\right\} \\
& =\int\left\{\mathbf{E}_{B} \wedge d \Lambda+d\left(e^{-2 \phi} \star H \wedge d \Lambda\right)\right\} .
\end{aligned}
$$

Integrating by parts the first term and using the Noether identity eq. (3.4c)

$$
\delta_{\Lambda} S=\int d\left(\Lambda \wedge \mathbf{E}_{B}+e^{-2 \phi} \star H \wedge d \Lambda\right) \equiv \int d \mathbf{J}[\Lambda] .
$$

The invariance of the action under these gauge transformations indicates that the current $\mathbf{J}[\Lambda]$ must be locally exact, so that, locally, there is a $\mathbf{Q}[\Lambda]$ such that $\mathbf{J}[\Lambda]=d \mathbf{Q}[\Lambda]$. It is easy to see that

$$
\mathbf{Q}[\Lambda]=\Lambda \wedge\left(e^{-2 \phi} \star H\right)
$$

The conserved charge is given by the integral of the conserved $(d-2)$-form $\mathcal{Q}[\Lambda]$ over $(d-2)$-dimensional compact surfaces $\mathcal{S}_{d-2}$ for $\Lambda$ s that leave invariant the KR field $B \mathrm{~s}$. These are closed 1-forms. Following [6, 32], using the Hodge decomposition theorem, these closed 1-forms $\Lambda$ can be written as the sum of an exact and a harmonic form $\Lambda_{e}=d \lambda$ and $\Lambda_{h}$, respectively. The exact form $\Lambda_{e}$ will not contribute to the integral on-shell because

$$
Q\left(\Lambda_{e}\right)=\int_{\mathcal{S}_{d-2}} d \lambda \wedge\left(e^{-2 \phi} \star H\right)=\int_{\mathcal{S}_{d-2}} d\left[\lambda \wedge\left(e^{-2 \phi} \star H\right)\right]-\int_{\mathcal{S}_{d-2}} \lambda \wedge \mathbf{E}_{B} .
$$

Therefore,

$$
Q(\Lambda)=\int_{\mathcal{S}_{d-2}} \Lambda_{h} \wedge\left(e^{-2 \phi} \star H\right)
$$

Then, using the duality between homology and cohomology, if $C_{\Lambda_{h}}$ is the $(d-3)$-cycle dual to $\Lambda_{h}$, we arrive at the charges

$$
Q(\Lambda)=-\frac{g_{s}^{(d) 2}}{16 \pi G_{N}^{(d)}} \int_{C_{\Lambda_{h}}} e^{-2 \phi} \star H,
$$

where we have added a conventional sign and recovered the factor of $g_{s}^{(d) 2}\left(16 \pi G_{N}^{(d)}\right)^{-1}$ that we have omitted. From the string theory point of view, these charges are just winding numbers of strings whose transverse space is the cycle $C_{\Lambda_{h}}$. Two homologically equivalent 
cycles give the same value of the charge on-shell, that is, if there are no sources of the KR field in the $(d-2)$-dimensional volume whose boundary is the union of the two properly oriented $(d-3)$-cycles.

Let us now consider the conserved charges associated to the invariance under $\delta_{\chi}$. This transformation acts on the 1-forms $\mathcal{A}^{I}$ and on the KR 2-form $B$. Transformations with constant $\chi^{I}$ (closed 0 -forms) leave invariant the 1-forms, but they do not leave invariant $B$. They only change it by an exact 2 -form $d\left(\frac{1}{2} \chi_{I} \mathcal{A}^{I}\right)$. Thus, we must add a compensating $\Lambda$ gauge transformation with parameter $\Lambda_{\chi}=-\frac{1}{2} \chi_{I} \mathcal{A}^{I}$ and consider the transformation of $B$

$$
\delta_{\chi} B=-\frac{1}{2} d\left(\chi_{I} \mathcal{A}^{I}\right)+\frac{1}{2} \chi_{I} d \mathcal{A}^{I}=-\frac{1}{2} d \chi_{I} \wedge \mathcal{A}^{I} .
$$

Then, from eqs. (2.13) and (2.16) and the modified transformation rule eq. (3.11), we get

$$
\begin{aligned}
\delta_{\chi} S= & \int\left\{\mathbf{E}_{B} \wedge \delta_{\chi} B+\mathbf{E}_{I} \wedge \delta_{\chi} \mathcal{A}^{I}\right. \\
& \left.+d\left[e^{-2 \phi} M_{I J} \star \mathcal{F}^{J} \wedge \delta_{\chi} \mathcal{A}^{I}+e^{-2 \phi} \star H \wedge\left(\delta_{\chi} B+\frac{1}{2} \mathcal{A}_{I} \wedge \delta_{\chi} \mathcal{A}^{I}\right)\right]\right\}, \\
= & \int\left\{\left(\mathbf{E}_{I}+\frac{1}{2} \mathbf{E}_{B} \wedge \mathcal{A}_{I}\right) \wedge d \chi^{I}+d\left[\left(e^{-2 \phi} M_{I J} \star \mathcal{F}^{J}+e^{-2 \phi} \star H \wedge \mathcal{A}_{I}\right) \wedge d \chi^{I}\right]\right\} .
\end{aligned}
$$

Integrating by parts the first term and using the Noether identities eqs. (3.4b) and (3.4c) we get

$$
\delta_{\chi} S=\int d\left\{(-1)^{d-1} \chi^{I}\left(\mathbf{E}_{I}+\frac{1}{2} \mathbf{E}_{B} \wedge \mathcal{A}_{I}\right)+\left(e^{-2 \phi} M_{I J} \star \mathcal{F}^{J}+e^{-2 \phi} \star H \wedge \mathcal{A}_{I}\right) \wedge d \chi^{I}\right\} .
$$

The usual argument leads to the conserved $(d-2)$-form

$$
\mathbf{Q}[\chi]=(-1)^{d} \chi^{I}\left(e^{-2 \phi} M_{I J} \star \mathcal{F}^{J}+e^{-2 \phi} \star H \wedge \mathcal{A}_{I}\right),
$$

and the definition of electric charges

$$
\mathcal{Q}_{I}=\frac{(-1)^{d-1} g_{s}^{(d) 2}}{16 \pi G_{N}^{(d)}} \int_{\mathcal{S}_{(d-2)}}\left(e^{-2 \phi} M_{I J} \star \mathcal{F}^{J}+e^{-2 \phi} \star H \wedge \mathcal{A}_{I}\right),
$$

where we have added a conventional sign. Again, this charge is on-shell invariant under homologically-equivalent deformations of $\mathcal{S}_{(d-2)}$. This follows from the equation of motion written in the alternative form eq. (2.17). It is also on-shell invariant under the $\delta_{\chi}$ transformations, in spite of the explicit occurrence of the vector fields $\mathcal{A}_{I}$ : the second term in the integrand has the same structure as the integrand of the KR charge and, for the same reason, it is invariant on-shell when we add to $\mathcal{A}_{I}$ exact 1 -forms.

This charge is, in the terminology used by Marolf in ref. [36], a Page charge but, as we have explained, apart from localized and conserved, it is also gauge invariant on-shell. The formalism leads us to use precisely this charge, which will be the one occurring in the first law of black hole mechanics.

Finally, let us consider the charge associated to the invariance under local Lorentz transformations $\delta_{\sigma}$, which act on the Vielbein and on all the fields derived from it: spin 
connection and curvature. Let us postpone for the time being the conditions that the parameters that leave all of them invariant have to satisfy and lets study the transformation of the action. From eqs. (2.13) and (2.16) we find

$$
\delta_{\sigma} S=\int\left\{\mathbf{E}_{a} \wedge \delta_{\sigma} e^{a}+d\left[-e^{-2 \phi} \star\left(e^{a} \wedge e^{b}\right) \wedge \delta_{\sigma} \omega_{a b}+2 \imath_{a} d e^{-2 \phi} \star\left(e^{a} \wedge e^{b}\right) \wedge \delta_{\sigma} e_{b}\right]\right\},
$$

and using eqs. (3.1a) and (3.2a) and the Noether identity eq. (3.4a), we find that the integrand immediately reduces to a total derivative,

$$
\begin{aligned}
\delta_{\sigma} S & =\int d \mathbf{J}[\sigma], \\
\mathbf{J}[\sigma] & =(-1)^{d-1} e^{-2 \phi} \mathcal{D} \sigma_{a b} \wedge \star\left(e^{a} \wedge e^{b}\right)+2 \sigma_{b c} \imath_{a} d e^{-2 \phi} \star\left(e^{a} \wedge e^{b}\right) \wedge e^{c} .
\end{aligned}
$$

The standard argument tells us that $\mathbf{J}[\sigma]=d \mathbf{Q}[\sigma]$. Integrating by parts the first term

$$
\left.\mathbf{J}[\sigma]=d\left\{(-1)^{d-1} e^{-2 \phi} \sigma_{a b} \star\left(e^{a} \wedge e^{b}\right)\right\}+3\left(\sigma_{[b c} \imath a\right] d e^{-2 \phi}\right) \star\left(e^{a} \wedge e^{b}\right) \wedge e^{c} .
$$

The last term vanishes identically because ${ }^{7} \star\left(e^{a} \wedge e^{b}\right) \wedge e^{c}=2 \eta^{c[a} \star e^{b]}$ and we arrive at

$$
\mathbf{Q}[\sigma]=(-1)^{d-1} e^{-2 \phi} \star\left(e^{a} \wedge e^{b}\right) \wedge \sigma_{a b} .
$$

Now we have to consider Lorentz parameters that leave all the fields invariant. The spin connection and curvature are left invariant by covariantly constant parameters

$$
\mathcal{D} \sigma^{a}{ }_{b}=0,
$$

but the invariance of the Vielbein $\sigma^{a}{ }_{b} e^{b}=0$ can only be satisfied for $\sigma^{a}{ }_{b}=0$, and would automatically imply the vanishing of $\mathbf{Q}[\sigma]$.

The $(d-2)$-form, though, reappears in the proof of the first law for a Lorentz parameter that is covariantly constant over the bifurcation surface. We also notice that terms of higher order in the Lorentz curvature, such as those which arise with $\alpha^{\prime}$ corrections, lead to a non-vanishing Lorentz charge ref. [41].

\subsection{Diffeomorphisms and covariant Lie derivatives}

As we have discussed in the introduction, out of the fundamental fields of our theory, only the dilaton $\phi$ and the $\mathrm{O}(n, n) /(\mathrm{O}(n) \times \mathrm{O}(n))$ scalars $\phi^{x}$ transform as a tensor under diffeomorphisms $\delta_{\xi} x^{\mu}=\xi^{\mu}$, that is ${ }^{8}$

$$
\begin{gathered}
\delta_{\xi} \phi=-£_{\xi} \phi=-\imath_{\xi} d \phi, \\
\delta_{\xi} \phi^{x}=-£_{\xi} \phi^{x}=-\imath_{\xi} d \phi^{x} .
\end{gathered}
$$

\footnotetext{
${ }^{7}$ Here we use the property

$$
\star \omega^{(p)} \wedge \hat{\xi}=\star \imath \xi \omega^{(p)},
$$

$$
\begin{aligned}
\delta_{\xi} g_{\mu \nu} & =-£_{\xi} g_{\mu \nu}=-2 \nabla_{(\mu} \xi_{\nu)}, \\
\delta_{\xi} \mathcal{F} & =-£_{\xi} \mathcal{F}=-\left(\imath_{\xi} d+d \imath_{\xi}\right) \mathcal{F}, \\
\delta_{\xi} H & =-£_{\xi} H=-\left(\imath_{\xi} d+d \imath_{\xi}\right) H .
\end{aligned}
$$
}

which is valid for any $p$-form $\omega^{(p)}$ and any vector field $\xi=\xi^{\mu} \partial_{\mu}$ and its dual 1-form $\hat{\xi}=\xi_{\mu} d x^{\mu}$.

${ }^{8}$ The metric $g_{\mu \nu}=\eta_{a b} e^{a}{ }_{\mu} e^{b}{ }_{\mu}$ and the 2- and 3-form field strengths $\mathcal{F}, H$ also transform as tensors: 
The Vielbein $e^{a}$, the vectors (1-forms), $\mathcal{A}$, and the KR 2-form, $B$, have gauge freedoms and transform as tensors up to compensating gauge transformations. These compensating gauge transformations can be determined by

1. Requiring gauge-covariance of the complete transformation law (which can then be interpreted as a gauge-covariant Lie derivative) and

2. Imposing that, for diffeomorphisms which are symmetries of the field configuration that we are considering (in particular, for isometries), the complete transformation (covariant Lie derivative) vanishes. The first condition ensures that this vanishing is gauge-invariant.

In what follows we will denote by $k$ the vector fields $\xi$ that generate diffeomorphisms that leave invariant the complete field configuration. $k$ is, in particular, a Killing vector of the metric.

In a recent paper [27] we reviewed the construction of a Lie derivative of the Vielbein, spin connection and curvature covariant under local Lorentz transformations (Lie-Lorentz derivative) of refs. $[9,10]$ that build upon earlier work by Lichnerowicz, Kosmann and others [12-15]. In ref. [27] we also dealt with Abelian vector fields in similar terms. It is convenient to quickly review these results starting with the Abelian vector case, adapted to the present situation.

The transformation of the Abelian vector fields $\mathcal{A}^{I}$ under diffeomorphisms can be defined as

$$
\delta_{\xi} \mathcal{A}^{I}=-\mathbb{L}_{\xi} \mathcal{A}^{I}
$$

where $\mathbb{L}_{\xi} \mathcal{A}^{I}$ is the Lie-Maxwell derivative, defined by

$$
\mathbb{L}_{\xi} \mathcal{A}^{I} \equiv \imath_{\xi} \mathcal{F}^{I}+d \mathcal{P}_{\xi}^{I}
$$

Here $\mathcal{P}_{\xi}{ }^{I}$ is a gauge-invariant $\mathrm{O}(n, n)$ vector of functions that depends on $\mathcal{A}^{I}$ and on the generator of diffeomorphisms $\xi$ and it is assumed to have the property that, when $\xi=k$, it satisfies the equation

$$
d \mathcal{P}_{k}^{I}=-\imath_{k} \mathcal{F}^{I}
$$

The invariance of the 2 -form $\mathcal{F}^{I}$ guarantees the local existence of $\mathcal{P}_{k}{ }^{I}$, which is known as the momentum map associated to $k$. On the other hand, eq. (3.26) ensures that the two properties of the variations of the fields under diffeomorphisms that we have demanded are satisfied. Finally, observe that the Lie-Maxwell derivative is just a combination of the standard Lie derivative plus a compensating gauge transformation with parameter

$$
\chi_{\xi}^{I}=\imath_{\xi} \mathcal{A}^{I}-\mathcal{P}_{\xi}^{I} .
$$

For fields with Lorentz indices (Vielbein, spin connection and curvature), the variation under diffeomorphisms is also given by (minus) a Lorentz-covariant generalization of the Lie derivative $\delta_{\xi}=-\mathbb{L}_{\xi}$ usually called Lie-Lorentz derivative refs. [9, 10, 12-15]. This derivative 
can also be constructed by adding to the standard Lie derivative a compensating Lorentz transformation with the parameter

$$
\sigma_{\xi}^{a b}=\imath_{\xi} \omega^{a b}-\nabla^{[a} \xi^{b]} .
$$

For the Vielbein, the Lie-Lorentz derivative can be expressed in several equivalent and manifestly Lorentz-covariant forms

$$
\begin{aligned}
\mathbb{L}_{\xi} e^{a}{ }_{\mu} & =\frac{1}{2} e^{a \nu}\left(\nabla_{\mu} \xi_{\nu}+\nabla_{\nu} \xi_{\mu}\right) \\
\mathbb{L}_{\xi} e^{a} & =\mathcal{D} \xi^{a}+P_{\xi}{ }^{a} e^{b}
\end{aligned}
$$

where

$$
P_{\xi}^{a b} \equiv \nabla^{[a} \xi^{b]},
$$

satisfies, when $\xi=k$, the equation

$$
\imath_{k} R^{a b}=-\mathcal{D} P_{k}^{a b},
$$

that shows that we can view $P_{k}{ }^{a b}$ as a momentum map as well. ${ }^{9}$

In the form eq. (3.29a) we immediately see that the Lie-Lorentz derivative of the Vielbein vanishes when $\xi=k$, a Killing vector. The same is true for the connection and curvature.

Observe that $P_{\xi}^{a b}$ transforms covariantly under local Lorentz transformations.

The above transformation of the Vielbein induce the following transformations of the spin connection and curvature that we quote for later use:

$$
\begin{aligned}
& \delta_{\xi} \omega^{a b}=-\mathbb{L}_{\xi} \omega^{a b}=-\left(\imath_{\xi} R^{a b}+\mathcal{D} P_{\xi}{ }^{a b}\right), \\
& \delta_{\xi} R^{a b}=-\mathbb{L}_{\xi} R^{a b}=-\left(\mathcal{D} \imath_{\xi} R^{a b}-2 P_{\xi}{ }^{[a}{ }_{c} R^{b] c}\right) .
\end{aligned}
$$

Observe that the Lie-Lorentz derivative of the spin connection has the same structure as that of the Abelian connection $\mathcal{A}^{I}$ in eq. (3.25), i.e. the inner product of $\xi$ with the curvature plus the derivative of the momentum map.

In asymptotically-flat stationary black-hole spacetimes with bifurcate horizon, if $k$ is the Killing vector whose Killing horizon coincides with the event horizon and $\mathcal{B H}$ is the bifurcation sphere,

$$
P_{k}^{a b}=\nabla^{[a} k^{b]} \stackrel{\mathcal{B H}}{=} \kappa n^{a b},
$$

where $\kappa$ is the surface gravity and $n^{a b}$ is the binormal to the event horizon, with the normalization $n^{a b} n_{a b}=-2$. The zeroth law of black-hole mechanics stating that $\kappa$ is constant over the horizon $[2,47]$ is associated to the Lorentz momentum map, just as the generalized zeroth law that states that the electric potential is also constant over the horizon in the Einstein-Maxwell theory is associated to the Maxwell momentum map [27]. ${ }^{10}$

\footnotetext{
${ }^{9}$ Compare this equation to eq. (3.26).

${ }^{10}$ This parallelism between zeroth laws was observed in [20], also in the wider context of Einstein-YangMills theories.
} 
We are going to see that further "generalized zeroth laws" are also associated to momentum maps when we restrict ourselves to the bifurcation surface. We will call them restricted generalized zeroth laws.

Let us now consider the KR field. It is convenient to start by considering the transformation of the 3-form field strength $H$ defined in eq. (2.10) under diffeomorphisms. Since it is gauge invariant, upon use of its Bianchi identity

$$
\delta_{\xi} H=-£_{\xi} H=-\imath_{\xi} d H-d \imath_{\xi} H=\imath_{\xi} \mathcal{F}_{I} \wedge \mathcal{F}^{I}-d \imath_{\xi} H .
$$

When $\xi=k$, this expression must vanish and we can use eq. (3.26), which leads to the identity

$$
\delta_{\xi} H=-d\left(\imath_{k} H+\mathcal{P}_{k I} \mathcal{F}^{I}\right)=0,
$$

which, in turn, implies the local existence of a gauge-invariant 1-form that we will also call a momentum map, satisfying

$$
-\imath_{k} H-\mathcal{P}_{k I} \mathcal{F}^{I}=d P_{k}
$$

The KR momentum map plays a fundamental role in the definition of the variation of the KR 2-form $B$ under diffeomorphisms which should be of the general form

$$
\delta_{\xi} B=-£_{\xi} B+\left(\delta_{\Lambda_{\xi}}+\delta_{\chi_{\xi}}\right) B
$$

where $\chi_{\xi}$ and $\Lambda_{\xi}$ are scalar and 1-form parameters of compensating gauge transformations. They will generically depend on $\mathcal{A}^{I}$ and $B$ as well as on $\xi$. $\chi_{\xi}^{I}$ has to be the same parameter used in the definition of the Lie-Maxwell derivative eq. (3.27) and we just have to determine $\Lambda_{\xi}$. Now, the Maxwell and Lorentz cases suggest that we try

$$
\Lambda_{\xi}=\imath_{\xi} B-P_{\xi}
$$

which leads to

$$
\begin{aligned}
\delta_{\xi} B & =-£_{\xi} B+d\left(\imath_{\xi} B-P_{\xi}\right)+\frac{1}{2} \chi_{\xi I} d \mathcal{A}^{I} \\
& =-\left(\iota_{\xi} H+\mathcal{P}_{\xi I} \mathcal{F}^{I}+d P_{\xi}\right)+\frac{1}{2} \mathcal{A}_{I} \wedge \imath_{\xi} \mathcal{F}^{I}+\frac{1}{2} \mathcal{P}_{\xi I} \mathcal{F}^{I} .
\end{aligned}
$$

When $\xi=k$, though,

$$
\delta_{k} B=d\left(\frac{1}{2} \mathcal{P}_{k I} \mathcal{A}^{I}\right)
$$

This is not zero but it can be absorbed into a redefinition of $\Lambda_{\xi}$ :

$$
\Lambda_{\xi}=\imath_{\xi} B-P_{\xi}-\frac{1}{2} \mathcal{P}_{k I} \mathcal{A}^{I}
$$

which gives the variation

$$
\delta_{\xi} B=-\left(\imath_{\xi} H+\mathcal{P}_{\xi I} \mathcal{F}^{I}+d P_{\xi}\right)-\frac{1}{2} \mathcal{A}_{I} \wedge \delta_{\xi} \mathcal{A}^{I} .
$$


This form of the variation makes it evident that $\delta_{k} B=0$, because $\delta_{k} \mathcal{A}^{I}=0$ and because of the definition of the KR momentum map 1-form eq. (3.36).

It remains to check that the vanishing of this variation is a gauge-invariant statement. Indeed, if we perform a gauge transformation in $\delta_{\xi} B$, taking into account that all the momentum maps and $\delta_{\xi} \mathcal{A}^{I}$ are gauge-invariant, we find

$$
\delta_{\text {gauge }} \delta_{\xi} B=-\frac{1}{2} \delta_{\text {gauge }} \mathcal{A}_{I} \wedge \delta_{\xi} \mathcal{A}^{I},
$$

which vanishes identically for $\xi=k$.

\subsection{The Wald-Noether charge}

The Wald-Noether charge is the conserved $(d-2)$-form associated to the invariance of the action under diffeomorphisms [1]. The transformations that we are going to consider (combinations of standard Lie derivative and gauge transformations, as we have explained) are

$$
\begin{aligned}
\delta_{\xi} \phi & =-\imath_{\xi} d \phi \\
\delta_{\xi} \phi^{x} & =-\imath_{\xi} d \phi^{x} . \\
\delta_{\xi} \mathcal{A}^{I} & =-\left(\imath_{\xi} \mathcal{F}^{I}+d \mathcal{P}_{\xi}{ }^{I}\right), \\
\delta_{\xi} e^{a} & =-\left(\mathcal{D} \xi^{a}+P_{\xi}{ }^{a} e^{b}\right), \\
\delta_{\xi} \omega^{a b} & =-\left(\imath_{\xi} R^{a b}+\mathcal{D} P_{\xi}{ }^{a b}\right), \\
\delta_{\xi} B+\frac{1}{2} \mathcal{A}_{I} \wedge \delta_{\xi} \mathcal{A}^{I} & =-\left(\imath_{\xi} H+\mathcal{P}_{\xi I} \mathcal{F}^{I}+d P_{\xi}\right) .
\end{aligned}
$$

From eq. (2.13), and using the definition of $\tilde{\mathbf{E}}_{I}$ in eqs. (2.15c) and (2.15d) to cancel the terms of the form $\mathbf{E}_{B} \wedge \mathcal{A}_{I} \wedge \delta_{\xi} \mathcal{A}^{I}$, we get

$$
\begin{aligned}
\delta_{\xi} S=-\int & \left\{\mathbf{E}_{a} \wedge\left(\mathcal{D} \imath_{\xi} e^{a}+P_{\xi}{ }^{a} e^{b}\right)+\mathbf{E}_{B} \wedge\left(\imath_{\xi} H+\mathcal{P}_{\xi I} \mathcal{F}^{I}+d P_{\xi}\right)\right. \\
& +\tilde{\mathbf{E}}_{I} \wedge\left(\imath_{\xi} \mathcal{F}^{I}+d \mathcal{P}_{\xi}{ }^{I}\right)+\mathbf{E}_{\phi} \imath_{\xi} d \phi+\mathbf{E}_{x} \imath_{\xi} d \phi^{x} \\
& \left.-d \Theta\left(\varphi, \delta_{\xi} \varphi\right)\right\}
\end{aligned}
$$

while, from eq. (2.16), we get

$$
\begin{aligned}
\Theta\left(\varphi, \delta_{\xi} \varphi\right)= & e^{-2 \phi} \star\left(e^{a} \wedge e^{b}\right) \wedge\left(\imath_{\xi} R_{a b}+\mathcal{D} P_{\xi a b}\right) \\
& -2 \imath_{a} d e^{-2 \phi} \star\left(e^{a} \wedge e^{b}\right) \wedge\left(\mathcal{D} \xi_{b}+P_{\xi b c} e^{c}\right) \\
& +8 e^{-2 \phi} \star d \phi \imath_{\xi} d \phi-e^{-2 \phi} g_{x y} \star d \phi^{y} \imath_{\xi} d \phi^{x} \\
& -e^{-2 \phi} M_{I J} \star \mathcal{F}^{J} \wedge\left(\imath_{\xi} \mathcal{F}^{I}+d \mathcal{P}_{\xi}^{I}\right) \\
& -e^{-2 \phi} \star H \wedge\left(\imath_{\xi} H+\mathcal{P}_{\xi I} \mathcal{F}^{I}+d P_{\xi}\right) .
\end{aligned}
$$


Next, we consider the terms in $\delta_{\xi} S$ that contain momentum maps, integrating by parts those which involve their derivatives:

$$
\begin{aligned}
\mathbf{E}_{a} \wedge & P_{\xi}{ }^{a} b e^{b}+\tilde{\mathbf{E}}_{I} \wedge d \mathcal{P}_{\xi}^{I}+\mathbf{E}_{B} \wedge\left(\mathcal{P}_{\xi I} \mathcal{F}^{I}+d P_{\xi}\right) \\
= & \mathbf{E}^{[a} \wedge e^{b]} P_{\xi a b}+P_{\xi} d \mathbf{E}_{B}+(-1)^{d} \mathcal{P}_{\xi I}\left[d \tilde{\mathbf{E}}^{I}+(-1)^{d} \mathbf{E}_{B} \wedge \mathcal{F}^{I}\right] \\
& +d\left(P_{\xi} \wedge \mathbf{E}_{B}+(-1)^{d-1} \mathcal{P}_{\xi}^{I} \tilde{\mathbf{E}}_{I}\right) .
\end{aligned}
$$

The terms in the first line vanish as a consequence of the Noether identities eqs. (3.4a)(3.4c) and we are left with the total derivative which will be added to $\Theta\left(\varphi, \delta_{\xi} \varphi\right)$. Thus, the variation of the action takes the form

$$
\begin{aligned}
& \delta_{\xi} S=-\int\left\{\mathbf{E}_{a} \wedge \mathcal{D} \imath_{\xi} e^{a}+\mathbf{E}_{B} \wedge \imath_{\xi} H+\tilde{\mathbf{E}}_{I} \wedge \imath_{\xi} \mathcal{F}^{I}+\mathbf{E}_{\phi} \imath_{\xi} d \phi+\mathbf{E}_{x} \imath_{\xi} d \phi^{x}\right. \\
&\left.-d\left[\boldsymbol{\Theta}\left(\varphi, \delta_{\xi} \varphi\right)-P_{\xi} \wedge \mathbf{E}_{B}+(-1)^{d} \mathcal{P}_{\xi}^{I} \tilde{\mathbf{E}}_{I}\right]\right\}
\end{aligned}
$$

Integrating the first term of eq. (3.48) by parts we get another total derivative to add to $\boldsymbol{\Theta}\left(\varphi, \delta_{\xi} \varphi\right)$ and $\left(\imath_{\xi} e^{a}=\xi^{a}\right)$

$$
(-1)^{d} \mathcal{D} \mathbf{E}_{a} \xi^{a}+\mathbf{E}_{B} \wedge \imath_{\xi} H+\tilde{\mathbf{E}}_{I} \wedge \imath_{\xi} \mathcal{F}^{I}+\mathbf{E}_{\phi} \imath_{\xi} d \phi+\mathbf{E}_{x} \imath_{\xi} d \phi^{x}=0,
$$

by virtue of the Noether identity associated to the invariance under diffeomorphisms and, therefore,

$$
\delta_{\xi} S=\int d \Theta^{\prime}\left(\varphi, \delta_{\xi} \varphi\right)
$$

where

$$
\boldsymbol{\Theta}^{\prime}\left(\varphi, \delta_{\xi} \varphi\right)=\boldsymbol{\Theta}\left(\varphi, \delta_{\xi} \varphi\right)+(-1)^{d} \mathbf{E}_{a} \xi^{a}-P_{\xi} \wedge \mathbf{E}_{B}+(-1)^{d} \mathcal{P}_{\xi}^{I} \tilde{\mathbf{E}}_{I}
$$

Usually, the last three terms, which are proportional to equations of motion and vanish on-shell, are ignored for this very reason. However, we have found that keeping them is actually quite useful for finding the Wald-Noether charge, because they are exactly what is needed to write $\mathbf{J}$ as a total derivative. Without them, we would have had to guess which combinations of the equations of motion should be added to achieve that goal. Furthermore, the result that we will obtain will be valid off-shell.

Since the action is exactly invariant under the gauge transformations eq. (3.1), but it is only invariant up to a total derivative under standard infinitesimal diffeomorphisms, under the combined transformations eqs. (3.44)

$$
\delta_{\xi} S=-\int d l_{\xi} \mathbf{L}
$$

which, combined with eq. (3.50), leads to the identity

$$
d \mathbf{J}=0
$$

which holds off-shell for arbitrary $\xi$ with

$$
\mathbf{J} \equiv \boldsymbol{\Theta}^{\prime}\left(\varphi, \delta_{\xi} \varphi\right)+\imath_{\xi} \mathbf{L} .
$$


Eq. (3.53) implies the local existence of a $(d-2)$-form $\mathbf{Q}[\xi]$ such that

$$
\mathbf{J}=d \mathbf{Q}[\xi]
$$

Using the previous results we find that, up to total derivatives and up to the overall factor $g_{s}^{(d) 2}\left(16 \pi G_{N}^{(d)}\right)^{-1}$ that we are suppressing to get simpler expressions

$$
\begin{aligned}
\mathbf{Q}[\xi]= & (-1)^{d} \star\left(e^{a} \wedge e^{b}\right)\left[e^{-2 \phi} P_{\xi a b}-2 \imath_{a} d e^{-2 \phi} \xi_{b}\right] \\
& +(-1)^{d-1} \mathcal{P}_{\xi}{ }^{I}\left(e^{-2 \phi} M_{I J} \star \mathcal{F}^{J}\right)-P_{\xi} \wedge\left(e^{-2 \phi} \star H\right) .
\end{aligned}
$$

\section{Zeroth laws}

The zeroth law and its generalizations, ensuring that the surface gravity and the electrostatic potential are constant over the event (Killing) horizon $\mathcal{H}$ are important ingredients in the standard derivation of the first law of black-hole mechanics in the context of the Einstein-Maxwell theory [2]. In presence of higher-rank $p$-form fields, it is not clear how these laws should be further generalized. However, it is possible to proof the first law using Wald's formalism working on the bifurcation sphere $\mathcal{B H}$, where the Killing vector $k$ associated to the horizon vanishes. This restricts the validity of the proof to bifurcate horizons but, on the other hand, it makes it possible to carry out the proof using a more restricted form of the (generalized) zeroth laws which states the closedness of the electrostatic potential and its higher-rank generalizations on $\mathcal{B H}$. Since the electrostatic potential is a scalar, its closedness implies that it is constant on $\mathcal{B H}$, which is a restricted version of the generalized zeroth law. For higher-rank potentials closedness is, actually, all we need, as we will see in the next section.

We start by assuming that all the field strengths of the theory are regular on the horizon. ${ }^{11}$ This implies that

$$
\begin{gathered}
\imath_{k} \mathcal{F}^{I} \stackrel{\mathcal{B H}}{=} 0 \\
\imath_{k} H \stackrel{\mathcal{B H}}{=} 0 .
\end{gathered}
$$

The first equation directly implies the closedness of the components of the momentum map $\mathcal{P}_{k}^{I}$ on $\mathcal{B H}$ on account of its definition eq. (3.26), and, hence, its constancy on $\mathcal{B H}$, a statement that we can call restricted generalized zeroth law after the natural identification of $\mathcal{P}_{k}^{I}$ with the electrostatic black-hole potential $\Phi^{I}$. Observe that, our gauge-invariant definition of the electrostatic black-hole potential guarantees that it is fully defined up to an additive constant that can be determined by setting the value of the potential at infinity to zero.

Using eq. (4.1b) and the constancy of $\mathcal{P}_{k}^{I}$ on $\mathcal{B H}$ in the definition of the KR momentum map eq. (3.36) we find that

$$
0 \stackrel{\mathcal{B H}}{=}-\imath_{k} H=d P_{k}+\mathcal{P}_{k I} \mathcal{F}^{I} \stackrel{\mathcal{H}}{=} d\left(P_{k}+\mathcal{P}_{k I} \mathcal{A}^{I}\right)
$$

\footnotetext{
${ }^{11}$ Observe that in this theory in which all the field strengths are gauge-invariant, this is a gauge-invariant statement that should be valid in a regular coordinate patch.
} 
We can call the combination $P_{k}+\mathcal{P}_{k I} \mathcal{A}^{I}$ that is closed on $\mathcal{B H}$ the KR black-hole potential $\Phi$ and its closedness can be understood as another restricted generalized zeroth law of black-hole mechanics in this theory. Observe that $\Phi$ is not gauge-invariant, but $P_{k}$ is only defined up to shifts by exact 1-forms anyway and, when we use $\Phi$ as the 1-form $\Lambda$ in the calculation of the KR charge eq. (3.9), the addition of exact 1-forms does not change the value of the associated KR charge eq. (3.10). The fact that this $\Phi$ occurs in the expressions leading to the first law precisely plays this role is quite a non-trivial check of the consistency of our results.

\section{The first law}

We start by defining the pre-symplectic $(d-1)$-form [48]

$$
\omega\left(\varphi, \delta_{1} \varphi, \delta_{2} \varphi\right) \equiv \delta_{1} \boldsymbol{\Theta}\left(\varphi, \delta_{2} \varphi\right)-\delta_{2} \boldsymbol{\Theta}\left(\varphi, \delta_{1} \varphi\right),
$$

and the symplectic form relative to the Cauchy surface $\Sigma$

$$
\Omega\left(\varphi, \delta_{1} \varphi, \delta_{2} \varphi\right) \equiv \int_{\Sigma} \omega\left(\varphi, \delta_{1} \varphi, \delta_{2} \varphi\right) .
$$

Now, following ref. [3], when $\varphi$ solves the equations of motion $\mathbf{E}_{\varphi}=0$ if $\delta_{1} \varphi=\delta \varphi$ is an arbitrary variation of the fields and $\delta_{2} \varphi=\delta_{\xi} \varphi$ is their variation under diffeomorphisms, we have that

$$
\omega\left(\varphi, \delta \varphi, \delta_{\xi} \varphi\right)=\delta \mathbf{J}+d \imath_{\xi} \boldsymbol{\Theta}^{\prime}=\delta d \mathbf{Q}[\xi]+d \imath_{\xi} \boldsymbol{\Theta}^{\prime},
$$

where, in our case, $\mathbf{J}=d \mathbf{Q}$, where $\mathbf{Q}$ is given by eq. (3.56) and $\boldsymbol{\Theta}^{\prime}$ is given in eq. (3.51). Since, on-shell, $\Theta=\boldsymbol{\Theta}^{\prime}$, we have that, if $\delta \varphi$ satisfies the linearized equations of motion, $\delta d \mathbf{Q}=d \delta \mathbf{Q}$. Furthermore, if the parameter $\xi=k$ generates a transformation that leaves invariant the field configuration, $\delta_{k} \varphi=0,{ }^{12}$ linearity implies that $\omega\left(\varphi, \delta \varphi, \delta_{k} \varphi\right)=0$, and

$$
d\left(\delta \mathbf{Q}[k]+\imath_{k} \mathbf{\Theta}^{\prime}\right)=0 .
$$

Integrating this expression over a hypersurface $\Sigma$ with boundary $\delta \Sigma$ and using Stokes' theorem we arrive at

$$
\int_{\delta \Sigma}\left(\delta \mathbf{Q}[k]+\imath_{k} \mathbf{\Theta}^{\prime}\right)=0
$$

We are interested in asymptotically flat, stationary, black-hole spacetimes and we choose $k$ as the Killing vector whose Killing horizon coincides with the event horizon $\mathcal{H}$, which we assume to be a bifurcate horizon. This Killing vector $k$ is assumed to be linear combination with constant coefficients $\Omega^{n}$ of the timelike Killing vector associated to stationarity, $t^{\mu} \partial_{\mu}$ and the $\left[\frac{1}{2}(d-1)\right]$ inequivalent rotations $\phi_{n}^{\mu} \partial_{\mu}$

$$
k^{\mu}=t^{\mu}+\Omega^{n} \phi_{n}^{\mu} .
$$

Furthermore, we choose the hypersurface $\Sigma$ to be the space between infinity and the bifurcation sphere $(\mathcal{B H})$ on which $k=0$. Then, its boundary $\delta \Sigma$ has two disconnected pieces: a

\footnotetext{
${ }^{12}$ We have constructed variations of the fields $\delta_{\xi}$ for which this is possible.
} 
$(d-2)$-sphere at infinity, $\mathrm{S}_{\infty}^{d-2}$, and the bifurcation sphere $\mathcal{B H}$. Then, taking into account that $k=0$ on $\mathcal{B H}$, we obtain the relation

$$
\delta \int_{\mathcal{B H}} \mathbf{Q}[k]=\int_{\mathbf{S}_{\infty}^{d-2}}\left(\delta \mathbf{Q}[k]+\imath_{k} \mathbf{\Theta}^{\prime}\right) .
$$

As explained in refs. $[3,6]$, the right-hand side can be identified with $\delta M-\Omega^{m} \delta J_{n}$, where $M$ is the total mass of the black-hole spacetime and $J_{n}$ are the independent components of the angular momentum.

Using the explicit form of $\mathbf{Q}[k]$, eq. (3.56), and restoring the overall factor $g_{s}^{(d) 2}\left(16 \pi G_{N}^{(d)}\right)^{-1}$, we find

$$
\begin{aligned}
\delta \int_{\mathcal{B H}} \mathbf{Q}[k]= & \frac{(-1)^{d-1} g_{s}^{(d) 2}}{16 \pi G_{N}^{(d)}} \delta \int_{\mathcal{B H}} \mathcal{P}_{k}^{I}\left(e^{-2 \phi} M_{I J} \star \mathcal{F}^{J}\right) \\
& -\frac{g_{s}^{(d) 2}}{16 \pi G_{N}^{(d)}} \delta \int_{\mathcal{B H}} P_{k} \wedge\left(e^{-2 \phi} \star H\right) \\
& +\frac{(-1)^{d} g_{s}^{(d) 2}}{16 \pi G_{N}^{(d)}} \delta \int_{\mathcal{B H}} \star\left(e^{a} \wedge e^{b}\right)\left[e^{-2 \phi} P_{k a b}-2 \imath_{a} d e^{-2 \phi} k_{b}\right] .
\end{aligned}
$$

The last term vanishes over the bifurcation sphere and will be removed from now on.

As it is, this expression has two problems that make it difficult for us to obtain the kind of terms that occur in the first law. In the first line, we have an expression that we should be able to interpret in terms of the electric charges $\mathcal{Q}_{I}$. However, when we compare this with eq. (3.15) we see that the second term in the integrand is missing. Without that term, the charge is not conserved. On the other hand, in the second line, we have an expression that we should be able to interpret in terms of the KR charge using eq. (3.9). However, the 1 -form $P_{k}$ is not closed on $\mathcal{B H}$.

The solution to these two problems is unique: the addition and subtraction of the term $\mathcal{P}_{k I} \mathcal{A}^{I} \wedge\left(e^{-2 \phi} \star H\right)$ in the integrand, so that the integral to evaluate on $\mathcal{B H}$ takes the form

$$
\begin{aligned}
\delta \int_{\mathcal{B H}} \mathbf{Q}[k]= & \frac{(-1)^{d-1} g_{s}^{(d) 2}}{16 \pi G_{N}^{(d)}} \delta \int_{\mathcal{B H}} \mathcal{P}_{k}^{I}\left[e^{-2 \phi} M_{I J} \star \mathcal{F}^{J}+e^{-2 \phi} \star H \wedge \mathcal{A}_{I}\right] \\
& -\frac{g_{s}^{(d) 2}}{16 \pi G_{N}^{(d)}} \delta \int_{\mathcal{B H}}\left(P_{k}+\mathcal{P}_{k I} \mathcal{A}^{I}\right) \wedge\left(e^{-2 \phi} \star H\right) \\
& +\frac{(-1)^{d} g_{s}^{(d) 2}}{16 \pi G_{N}^{(d)}} \delta \int_{\mathcal{B H}} e^{-2 \phi} \star\left(e^{a} \wedge e^{b}\right) P_{k a b} .
\end{aligned}
$$

Now, using the generalized zeroth law that ensures that $\mathcal{P}_{k}{ }^{I} \equiv \Phi^{I}$ is constant over $\mathcal{H}$, in particular on $\mathcal{B H}$, and the definition of electric charge eq. (3.15), the first term in the right-hand side takes the form

$$
\Phi^{I} \delta \mathcal{Q}_{I}
$$

Next, from the closedness of the combination $\Phi=P_{k}+\mathcal{P}_{k I} \mathcal{A}^{I}$ on $\mathcal{B H}$, (the restricted generalized zeroth law) using the Hodge decomposition

$$
P_{k}+\mathcal{P}_{k I} \mathcal{A}^{I} \stackrel{\mathcal{B H}}{=} d e+\Phi^{i} \Lambda_{h i},
$$


where the $\Lambda_{h i}$ are harmonic 1-forms on $\mathcal{B H}$ and the $\Phi^{i}$ are constants that have the interpretation of potentials associated to the charge of the KR field (the dipole charge of ref. [37] in particular), and using the definition eq. (3.10), we find that the second term in the right-hand side takes the form

$$
\Phi^{i} \delta Q_{i}, \quad Q_{i} \equiv Q\left[\Lambda_{h i}\right] .
$$

Observe that the addition and subtraction of the term $\mathcal{P}_{k I} \mathcal{A}^{I} \wedge\left(e^{-2 \phi} \star H\right)$ has been crucial to recover the correct definition of the charges which, in particular, demands the occurrence of the closed 1-form $P_{k}+\mathcal{P}_{k} \mathcal{A}^{I}$.

Now, let us consider the third integral. Before we compute it explicitly, we notice that the integrand is identical, up to a sign, to the Lorentz charge eq. (3.20) computed for the Lorentz parameter $P_{k}{ }_{b}{ }_{b}$ which is covariantly constant over the bifurcation surface. This coincidence is very intriguing and will be further explored in ref. [41].

Using eq. (3.33)

$$
\begin{aligned}
\frac{(-1)^{d} \kappa}{16 \pi G_{N}^{(d)}} \delta \int_{\mathcal{B H}} e^{-2\left(\phi-\phi_{\infty}\right)} \star\left(e^{a} \wedge e^{b}\right) n_{a b} & =-\frac{\kappa}{16 \pi G_{N}^{(d)}} \delta \int_{\mathcal{B H}} e^{-2\left(\phi-\phi_{\infty}\right)} n^{a b} n_{a b} \\
& =T \delta \frac{\mathcal{A}_{\mathcal{H}}}{4 G_{N}^{(d)}}
\end{aligned}
$$

where we have used the normalization of the binormal $n_{a b} n^{a b}=-2, T=\kappa / 2 \pi$ is the Hawking temperature and

$$
\mathcal{A}_{\mathcal{H}} \equiv \int_{\mathcal{B}} d^{d-2} S e^{-2\left(\phi-\phi_{\infty}\right)}
$$

is the area of the horizon measured with the modified Einstein frame metric [49] which is obtained from the string one by multiplying by the conformal factor $e^{-4\left(\phi-\phi_{\infty}\right) /(d-2)}$, and computed using the spatial section $\mathcal{B H}$.

We finally get the following expression for the first law of black hole mechanics in the Heterotic Superstring effective action to leading order in $\alpha^{\prime}$ :

$$
\delta M=T \delta \frac{\mathcal{A}_{\mathcal{H}}}{4 G_{N}^{(d)}}+\Omega^{m} \delta J_{m}+\Phi^{i} \delta Q_{i}+\Phi^{I} \delta \mathcal{Q}_{I},
$$

which leads to the interpretation of the area of the horizon divided by $4 G_{N}^{(d)}$ as the blackhole entropy.

\section{Momentum maps for black rings in $d=5$}

In this section we are going to illustrate how the definitions made and the properties proven in the previous sections work in an explicit example. In particular, we are going to determine the values of the momentum maps, checking the restricted generalized zeroth laws.

The solution we are going to consider is a non-extremal, charged, black ring solution of pure $\mathcal{N}=1, d=5$ supergravity which can be easily embedded in the toroidallycompactified Heterotic Superstring effective field theory using the results in appendix A. 
This embedding is necessary because all the definitions and formulae that we have developed are adapted to that theory. In appendix A we show how the action eq. (2.4), for $d=5$ can be consistently truncated to that of pure $\mathcal{N}=1, d=5$ supergravity eq. (A.26) in two steps:

1. A direct truncation of some fields of the Heterotic theory, to obtain a model of $\mathcal{N}=1, d=5$ supergravity coupled to two vector multiplets. The Kalb-Ramond 2-form has to be dualized into a 1-form in order to obtain the supergravity theory in the standard form, with 31 -forms which can be treated on the same footing and which may be linearly combined.

2. A consistent truncation of the two vector supermultiplets. In this truncation, rather than setting two of the vector fields to zero, they are identified with the surviving vector, up to numerical factors. This allows the scalars in the vector supermultiplets to take their vacuum values.

Given a solution of pure $\mathcal{N}=1, d=5$ supergravity, one can easily retrace those steps, restoring, first, the two "matter" vector fields so the solution becomes now a solution of $\mathcal{N}=1, d=5$ supergravity coupled to two vector multiplets. Then, dualizing the vector in the supergravity multiplet to recover the Kalb-Ramond 2-form, the solution can immediately be interpreted as a solution of the Heterotic Superstring effective field theory in which many other fields simply take their vacuum values.

The non-extremal, charged, black ring solution that we are going to consider is the one given in section 4 of ref. [46]. This solution belongs to a more general family of nonsupersymmetric black rings with three charges $\alpha_{i}$, three dipoles $\mu_{i}$, with $i=1,2,3$, and two angular momenta $J_{\varphi}$ and $J_{\psi}$ in the theory with two vector supermultiplets. The solution above corresponds to setting all three charges and three dipoles equal, $\alpha_{i}=\alpha$ and $\mu_{i}=\mu$ for all $i$. This identification of the charges and dipoles coprresponds to the identification between the vector fields that leads from the supergravity theory with matter to the theory of pure supergravity. Let us review the solution and its main features.

The physical fields of the solution (the metric and the Abelian connection $A$ ) can be written in terms of the five parameters $(R, \alpha, \mu, \lambda, \nu)$ (all of them dimensionless except for the length scale $R$ ) and the three functions, $F(\xi), H(\xi)$ and $G(\xi)$, given by

$$
H(\xi)=1-\mu \xi, \quad F(\xi)=1+\lambda \xi, \quad G(\xi)=\left(1-\xi^{2}\right)(1+\nu \xi) .
$$

The line element is

$$
\begin{aligned}
d s^{2}= & \frac{U(x, y)}{h_{\alpha}^{2}(x, y)}\left(d t+\omega_{\psi}(y) d \psi+\omega_{\varphi}(x) d \varphi\right)^{2}-h_{\alpha}(x, y) F(x) H(x) H(y)^{2} \times \\
& \times \frac{R^{2}}{(x-y)^{2}}\left[-\frac{G(y)}{F(y) H(y)^{3}} d \psi^{2}-\frac{d y^{2}}{G(y)}+\frac{d x^{2}}{G(x)}+\frac{G(x)}{F(x) H(x)^{3}} d \varphi^{2}\right],
\end{aligned}
$$

where we use the shorthand notation $s=\sinh \alpha$ and $c=\cosh \alpha$, the following combinations of the fundamental parameters

$$
C_{\lambda}=\epsilon_{\lambda} \sqrt{\lambda(\lambda-\nu) \frac{1+\lambda}{1-\lambda}}, \quad C_{\mu}=\epsilon_{\mu} \sqrt{\mu(\mu+\nu) \frac{1-\mu}{1+\mu}}, \quad \epsilon_{\lambda, \mu}= \pm 1,
$$


and the following combinations of the fundamental functions in eq. (6.1)

$$
\begin{aligned}
U(x, y) & =\frac{H(x)}{H(y)} \frac{F(y)}{F(x)}, \\
h_{\alpha}(x, y) & =1+\frac{(\lambda+\mu)(x-y)}{F(x) H(y)} s^{2}, \\
\omega_{\psi}(y) & =R(1+y)\left[\frac{1}{F(y)} C_{\lambda} c^{3}-\frac{3}{H(y)} C_{\mu} c s^{2}\right], \\
\omega_{\varphi}(x) & =-R(1+x) s\left(\frac{1}{F(x)} C_{\lambda} s^{2}-\frac{3}{H(x)} C_{\mu} c^{2}\right) .
\end{aligned}
$$

Finally, the gauge field reads

$$
\begin{aligned}
-A / \sqrt{3}= & \frac{U(x, y)-1}{h_{\alpha}(x, y)} c s d t \\
& +\frac{R(1+y)}{h_{\alpha}(x, y)}\left[\frac{U(x, y)}{F(y)} C_{\lambda} c^{2} s-\frac{U(x, y)}{H(y)} C_{\mu} s^{3}-\frac{2}{H(y)} C_{\mu} c^{2} s\right] d \psi \\
& +\frac{R(1+x)}{h_{\alpha}(x, y)}\left[2 \frac{U(x, y)}{H(x)} C_{\mu} c s^{2}-\frac{1}{F(x)} C_{\lambda} c s^{2}+\frac{1}{H(x)} C_{\lambda} c^{3}\right] d \varphi .
\end{aligned}
$$

The parameters of the solution must satisfy the constraints

$$
0<\nu \leq \lambda<1, \quad 0 \leq \mu<1,
$$

to avoid naked singularities. Additional constraints arise from the condition of absence of Dirac-Misner strings and conical sigularities, as we are going to see.

The coordinates $x, y$ take values in

$$
-\infty<y \leq-1, \quad-1 \leq x \leq 1 .
$$

The surfaces of constant $y$ have the topology $\mathrm{S}^{2} \times \mathrm{S}^{1} . x$ is a polar coordinate on the $\mathrm{S}^{2}$ (essentially, $x \sim \cos \theta$ ), which is also parametrized by $\varphi$, which plays the role of azymuthal angle. $\psi$ parametrizes the $\mathrm{S}^{1}$, see figure 1 . Spatial infinity is approached when both $x$ and $y$ go to -1 , although the coordinates are ill-defined in that limit. ${ }^{13}$ The orbits of the vector $\partial_{\varphi}$ close off at $x=-1$, but do not do the same at $x=1$ unless $\omega_{\varphi}(x=+1)=0$, which can forces us to require

$$
\frac{C_{\lambda}}{1+\lambda} s^{2}=\frac{3 C_{\mu}}{1-\mu} c^{2}
$$

which removes any possible Dirac-Misner strings. (The same constraint makes $A_{\varphi}(x=+1)$ independent of $y$.) Then, the fixed point sets of $\partial_{\psi}$ and $\partial_{\varphi}$ are, respectively, $y=-1$ (axis of the ring) and $x=1,-1$ (inner and outer axes of the $\mathrm{S}^{2}$ ).

Finally, the periods of $\psi$ and $\varphi$ must be chosen appropriately so as to avoid conical singularities. The axes $y=-1$ and $x=-1$ (which extend to infinity) are regular for the periods

$$
\Delta \psi=\Delta \varphi=2 \pi \frac{\sqrt{1-\lambda}}{1-\nu}(1+\mu)^{3 / 2}
$$

\footnotetext{
${ }^{13}$ Good coordinates at infinity can be found in ref. [46].
} 


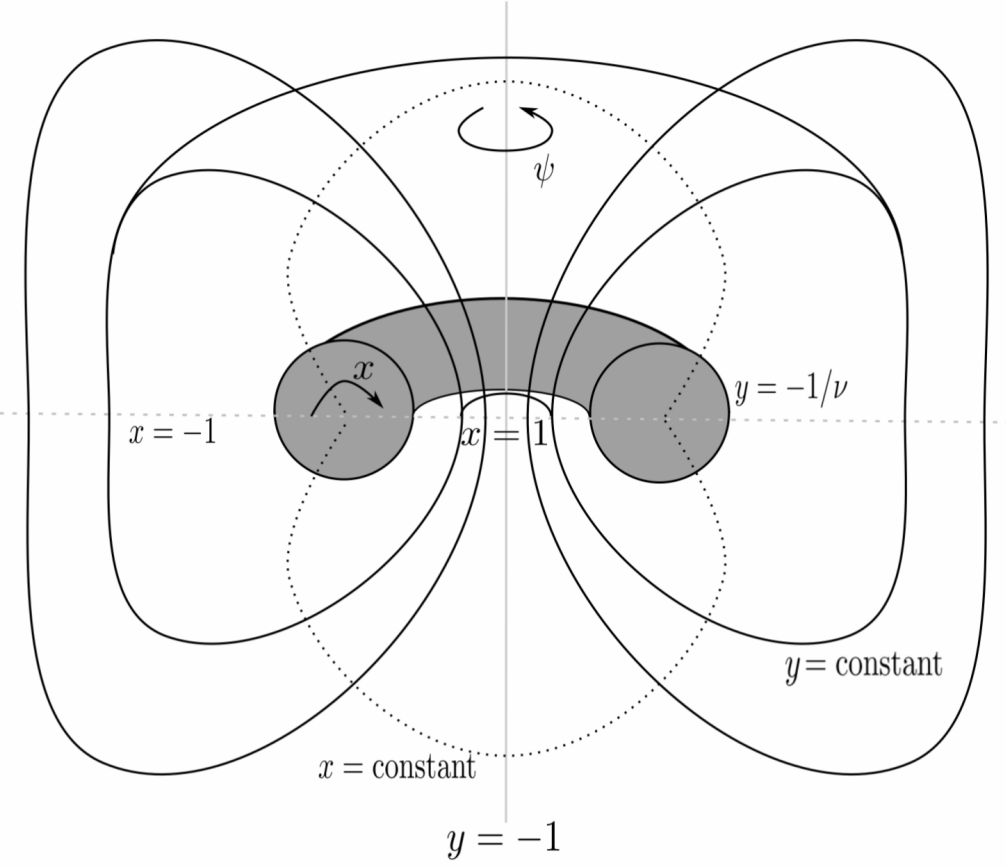

Figure 1. Sketch of a section of constant $t$ and $\varphi$ of the black ring (figure based on ref. [37]). The disc at $x=1$ and infinite annulus at $x=-1$ are the axes (fixed points) of $\partial_{\varphi}$, while the axis of the ring is at $y=-1$ (fixed points of $\partial_{\psi}$ ). Surfaces of constant $y$ have topology $S^{1} \times S^{2} . y=-1 / \nu$ corresponds to the horizon (shaded surface) while surfaces of constant $y \in(-1 / \nu,-1)$ are fatter rings containing the horizon in their interior.

For generic values of the parameters, though, the period of $\varphi$ required by smoothness at the inner axis, $x=1$, differs from the above $\Delta \varphi$. Making both periods coincide ("balancing" the ring) is possible only when the following constraint holds

$$
\left(\frac{1-\nu}{1+\nu}\right)^{2}=\frac{1-\lambda}{1+\lambda}\left(\frac{1+\mu}{1-\mu}\right)^{3}
$$

Henceforth we shall assume that eqs. (6.8) and (6.10) hold, so that, effectively, we will be dealing with a three-parameter family of solutions. As shown in ref. [46], the mass, the two independent angular momenta and the area of the event horizon of the solution read

$$
\begin{aligned}
M & =\frac{3 \pi R^{2}}{4 G_{N}^{(5)}} \frac{(\lambda+\mu)(1+\mu)^{2}}{1-\nu} \cosh 2 \alpha \\
J_{\psi} & =\frac{\pi R^{3}}{2 G_{N}^{(5)}} \frac{(1-\lambda)^{3 / 2}(1+\mu)^{9 / 2}}{(1-\nu)^{2}}\left[\frac{C_{\lambda}}{1-\lambda} c^{3}-\frac{3 C_{\mu}}{1+\mu} s^{2} c\right] \\
J_{\varphi} & =-\frac{3 \pi R^{3}}{G_{N}^{(5)}} \frac{\sqrt{1-\lambda}(1+\mu)^{7 / 2}(\lambda+\mu)}{(1-\nu)^{2}(1-\mu)} C_{\mu} c^{2} s, \\
\mathcal{A}_{\mathcal{H}} & =8 \pi^{2} R^{3} \frac{(1-\lambda)(\lambda-\nu)^{1 / 2}(1+\mu)^{3}(\nu+\mu)^{3 / 2}}{(1-\nu)^{2}(1+\nu)}\left|\frac{C_{\lambda}}{\lambda-\nu} c^{3}+\frac{3 C_{\mu}}{\nu+\mu} s^{2} c\right| .
\end{aligned}
$$


There is an ergosurface at $y=-1 / \lambda$, where the norm of $\partial_{t}$ vanishes, and the event horizon lies at $y=-1 / \nu$. It is a Killing horizon of

$$
k=\partial_{t}+\Omega \partial_{\psi},
$$

where $\Omega$, the angular velocity of the horizon in the direction $\psi$, can be conveniently written as $\Omega=-1 / \omega_{\psi}(-1 / \nu) .{ }^{14}$ A rather unusual property of this solution is that the horizon has no angular velocity in the direction $\varphi$ even though $J_{\varphi} \neq 0$. Finally, the horizon temperature is

$$
T_{\mathcal{H}}^{-1}=4 \pi R \frac{\sqrt{\lambda-\nu}(\mu+\nu)^{3 / 2}}{\nu(1+\nu)}\left|\frac{C_{\lambda}}{\lambda-\nu} c^{3}+\frac{3 C_{\mu}}{\nu+\mu} s^{2} c\right| .
$$

This solution of pure $\mathcal{N}=1, d=5$ supergravity corresponds to a following solution of the Heterotic Superstring effective field theory compactified on $\mathrm{T}^{4} \times \mathrm{S}^{1}$ with the same metric and the non-trivial matter fields given by ${ }^{15}$

$$
\begin{aligned}
\phi & =\phi_{\infty} \\
M_{I J} & =\left(\begin{array}{cc}
k_{\infty}^{2} & 0 \\
0 & k_{\infty}^{-2}
\end{array}\right), \\
\mathcal{A}^{I} & =\left(\begin{array}{c}
k_{\infty}^{-1} \\
k_{\infty}
\end{array}\right) \mathcal{A}, \\
H & =d B-\frac{1}{2} \mathcal{A}_{I} \wedge \mathcal{F}^{I}=\star \mathcal{F}
\end{aligned}
$$

where, for convenience, we have introduced $\mathcal{A}=-A / \sqrt{3}$ and its field strength $\mathcal{F}=d \mathcal{A}$. Let us obtain the vector and KR momentum maps associated to the Killing vector $k$ in eq. (6.12) for this solution, denoted, respectively, as $\mathcal{P}_{k}{ }^{I}$ and $P_{k}$. In the following we consider a constant $t$ surface $\Sigma$ defined by which extends from the bifurcate surface (here, a ring) $\mathcal{B H}$ at $y=-1 / \nu$ to infinity (analogously to one leaf of the Einstein-Rosen bridge). The vector momentum maps $\mathcal{P}_{k}^{I}$ can be written as

$$
\mathcal{P}_{k}^{I}=\left(\begin{array}{c}
k_{\infty}^{-1} \\
k_{\infty}
\end{array}\right) \mathcal{P}_{k}
$$

where $\mathcal{P}_{k}$ satisfies the equation

$$
d \mathcal{P}_{k}=-\imath_{k} \mathcal{F} .
$$

Since in our gauge $£_{k} \mathcal{A}=0$ it is clear that a solution (as a matter of fact, any solution) of the above equation is provided by

$$
\mathcal{P}_{k}=\imath_{k} \mathcal{A}+C
$$

\footnotetext{
${ }^{14}$ Notice we work with coordinates $\varphi, \psi$ whose periods are not the standard ones, but those given in eq. (6.9).

${ }^{15}$ The fields that arise in the compactification over $\mathrm{T}^{4}$ and which are set to their vacuum values (they are trivial) have not been considered. In particular, the index $I$ takes only two values because the fields corresponding to the other values are trivial.
} 
for some constant $C$. Notice, though, that this is not the definition of the momentum map, but rather a particular form of $\mathcal{P}_{k}$ which is available in the gauge in which the black-ring solution is given. The momentum map is, by definition, gauge invariant. The constant $C$ is determined by demanding $\mathcal{P}_{k}$ (which will be interpreted as the black ring's electrostatic potential $\Phi$ ) to vanish at infinity, and it is not difficult to see that $C=0$.

This solution admits an analytic prolongation to the bifurcate ring $\mathcal{B H}$ at $y=-1 / \nu$ (and actually beyond that) and, in agreement with the generalised zeroth law, it is a constant over the whole event horizon $\mathcal{H}$ that we will denote by $\Phi_{\mathcal{H}}$

$$
\begin{aligned}
\mathcal{P}_{k} & \stackrel{\mathcal{H}}{=} \mathcal{P}_{k}(x,-1 / \nu) \\
& =-\frac{\cosh 2 \alpha\left[C_{\lambda}(\mu+\nu)+3 C_{\mu}(\lambda-\nu)\right]+C_{\lambda}(\mu+\nu)+C_{\mu}(\lambda-\nu)}{\cosh 2 \alpha\left[C_{\lambda}(\mu+\nu)+3 C_{\mu}(\lambda-\nu)\right]+C_{\lambda}(\mu+\nu)-3 C_{\mu}(\lambda-\nu)} \tanh \alpha \\
& \equiv \Phi_{\mathcal{H}} .
\end{aligned}
$$

Observe that, in the gauge in which the solution is given, the potential $\mathcal{A}$ is ill-defined over $\mathcal{B H}:{ }_{{ }_{k}} \mathcal{A}$ is a non-vanishing constant there and $k$ vanishes, which implies that $\mathcal{A}$ must diverge there. It is worth stressing that the momentum map is unaffected by such gauge pathologies since the solution eq. (6.17) extends from infinity all the way down to $\mathcal{B H}$ (and beyond). This is a consequence of the fact that, although the momentum maps may only exist locally, they are defined by a gauge invariant equation.

The KR momentum map 1-form, $P_{k}$, is defined by eq. (3.36), and, for this particular solution

$$
d P_{k}=-\left(\imath_{k} H+\mathcal{P}_{k}^{I} \mathcal{F}_{I}\right)=-\left(\imath_{k} \star \mathcal{F}+2 \mathcal{P}_{k} \mathcal{F}\right) .
$$

If we knew the KR potential $B$ in a gauge in which $£_{k} B=0$, using $\mathcal{P}_{k}=\imath_{k} \mathcal{A}$, we would obtain the KR momentum map 1-form

$$
P_{k}=\imath_{k} B-\mathcal{P}_{k} \mathcal{A}+\alpha,
$$

where $\alpha$ is an arbitrary closed 1 -form, $d \alpha=0$, that could be determined by imposing regularity: smoothness of $P_{k}$ both at the axis of the ring, $P_{\psi}(x, y=-1)=0$, and at the outer axis of the spheres, $P_{\varphi}(x=-1, y)=0$, so that it is well defined when approaching infinity). Finding $B$ is, however, as hard as finding $P_{k}$ directly from eq. (6.19), which is what we are going to do, taking into account that we are only interested in the pullback of $P_{k}$ to the constant- $t$ surface $\Sigma$, which must be of the form

$$
P_{k} \stackrel{\Sigma}{=} P_{k \varphi}^{\Sigma}(x, y) d \varphi+P_{k \psi}^{\Sigma}(x, y) d \psi,
$$

because of the general form of the solution.

The two functions $P_{k \varphi}^{\Sigma}(x, y)$ and $P_{k \psi}^{\Sigma}(x, y)$ are given by

$$
\begin{aligned}
P_{k \varphi}^{\Sigma}(x, y) & =-\int^{y}\left(\imath_{k} \star \mathcal{F}+2 \mathcal{P}_{k} \mathcal{F}\right)_{y \varphi} d y+f_{\varphi}(x) \\
& =-2 \mathcal{P}_{k} \mathcal{A}_{\varphi}+\int^{y} I_{\varphi}(x, y) d y+f_{\varphi}(x), \\
P_{k \psi}^{\Sigma}(x, y) & =-\int^{y}\left(\imath_{k} \star \mathcal{F}+2 \mathcal{P}_{k} \mathcal{F}\right)_{y \psi} d y+f_{\psi}(x) \\
& =-2 \mathcal{P}_{k} \mathcal{A}_{\psi}+\int^{y} I_{\psi}(x, y) d y+f_{\psi}(x),
\end{aligned}
$$


where

$$
\begin{aligned}
I_{\varphi}(x, y)= & 2 \mathcal{A}_{\varphi}\left(\partial_{y} \mathcal{A}_{t}+\Omega \partial_{y} \mathcal{A}_{\psi}\right) \\
& +\partial_{x} \mathcal{A}_{t}\left(\frac{R^{2} \Omega F(x) G(x) H(y) h(x, y)^{2}}{F(y) H(x)(x-y)^{2}}+\frac{F(y) G(x) H(y) \omega_{\psi}(y)\left(\Omega \omega_{\psi}(y)+1\right)}{F(x) G(y) H(x) h(x, y)}\right) \\
& -\partial_{x} \mathcal{A}_{t}\left(\frac{\Omega H(x)^{2} \omega_{\varphi}(x)^{2}}{H(y)^{2} h(x, y)}\right) \\
& -\partial_{x} \mathcal{A}_{\psi} \frac{F(y) G(x) H(y)\left(\Omega \omega_{\psi}(y)+1\right)}{F(x) G(y) H(x) h(x, y)}+\partial_{x} \mathcal{A}_{\varphi} \frac{\Omega H(x)^{2} \omega_{\varphi}(x)}{H(y)^{2} h(x, y)} \\
I_{\psi}(x, y)= & \frac{H(x)^{2}\left(\omega_{\varphi}(x) \partial_{x} \mathcal{A}_{t}-\partial_{x} \mathcal{A}_{\varphi}\right)}{H(y)^{2} h(x, y)}+2 \mathcal{A}_{\psi}\left(\partial_{y} \mathcal{A}_{t}+\Omega \partial_{y} \mathcal{A}_{\psi}\right),
\end{aligned}
$$

for some functions $f_{\varphi}(x)$ and $f_{\psi}(x)$ to be determined.

In this form, the functions are well defined at $y=-1 / \nu$ (and beyond), and we can analytically prolongate $P_{k}$ there.

The functions $f_{\varphi}(x)$ and $f_{\psi}(x)$ can be readily fixed from the fact that the combination $P_{k}+2 \mathcal{P}_{k} \mathcal{A}$ is closed on $\mathcal{B H}$ (the restricted generalized zeroth law). Indeed, pulling back on $\mathcal{B H}$ the KR momentum map eq. (6.19), one has

$$
d\left(P_{k}+2 \Phi_{\mathcal{H}} \mathcal{A}\right) \stackrel{\mathcal{B H}}{=} 0 .
$$

Thus, a solution of the form (6.21) that is well defined at $y=-1 / \nu$ must satisfy the boundary condition

$$
P_{k} \stackrel{\mathcal{B H}}{=}-2 \Phi_{\mathcal{H}} \mathcal{A}+C_{\varphi} d \varphi+C_{\psi} d \psi
$$

for some constants $C_{\varphi}$ and $C_{\psi}$. This implies that our solution reads

$$
\begin{gathered}
P_{k \varphi}^{\Sigma}(x, y)=-2 \mathcal{P}_{k} \mathcal{A}_{\varphi}+\int_{-1 / \nu}^{y} I_{\varphi}(x, y) d y+C_{\varphi}, \\
P_{\psi}^{\Sigma}(x, y)=-2 \mathcal{P}_{k} \mathcal{A}_{\psi}+\int_{-1 / \nu}^{y} I_{\psi}(x, y) d y+C_{\psi} .
\end{gathered}
$$

Remarkably,

$$
\begin{aligned}
\int_{-1 / \nu}^{y} I_{\varphi}(-1, y) d y= & 0, \quad \forall y \neq-1, \\
\int_{-1 / \nu}^{-1} I_{\psi}(x, y) d y= & \frac{\cosh 2 \alpha\left[C_{\lambda}(\mu+\nu)+C_{\mu}(\nu-\lambda)\right]+C_{\lambda}(\mu+\nu)+C_{\mu}(\lambda-\nu)}{\cosh 2 \alpha\left[C_{\lambda}(\mu+\nu)+3 C_{\mu}(\lambda-\nu)\right]+C_{\lambda}(\mu+\nu)-3 C_{\mu}(\lambda-\nu)} \times \\
& \times \frac{\nu-1}{\mu+\nu} C_{\mu} R \operatorname{sech} \alpha, \quad \forall x,
\end{aligned}
$$

so regularity at $y=-1$ and $x=-1$ is achieved by setting

$$
\begin{aligned}
C_{\varphi} & =0, \\
C_{\psi} & =\frac{\cosh 2 \alpha\left[C_{\lambda}(\mu+\nu)+C_{\mu}(\nu-\lambda)\right]+C_{\lambda}(\mu+\nu)+C_{\mu}(\lambda-\nu)}{\cosh 2 \alpha\left[C_{\lambda}(\mu+\nu)+3 C_{\mu}(\lambda-\nu)\right]+C_{\lambda}(\mu+\nu)-3 C_{\mu}(\lambda-\nu)} \frac{1-\nu}{\mu+\nu} C_{\mu} R \operatorname{sech} \alpha \\
& \equiv C(\lambda, \mu, \nu, \alpha) \frac{1-\nu}{\mu+\nu} C_{\mu} R \operatorname{sech} \alpha,
\end{aligned}
$$

which completes the solution. 
We conclude by noticing that the associated KR potential 1 -form at $\mathcal{B H}$ is purely harmonic and given by,

$$
\Phi_{K R}=P_{k}+2 \mathcal{P}_{k} \mathcal{A} \stackrel{\mathcal{B H}}{=} \Phi_{K R \tilde{\psi}} d \tilde{\psi},
$$

where $\tilde{\psi}=(2 \pi / \Delta \psi) \psi$ is the angular coordinate with canonical period $\tilde{\psi} \sim \tilde{\psi}+2 \pi$ and

$$
\Phi_{K R \tilde{\psi}}=C_{\psi} \frac{\Delta \psi}{2 \pi}=C(\lambda, \mu, \nu, \alpha) \frac{\sqrt{1-\lambda}(1+\mu)^{3 / 2}}{\mu+\nu} C_{\mu} R \operatorname{sech} \alpha .
$$

For $\alpha=0, \Phi_{K R}$ coincides with the potential given in ref. [37] up to (parameter-independent) numerical prefactors.

One could try to test the first law we have derived in this family of solutions. This calculation turns out to be algebraically very involved, as the area formula is not given directly in terms of the physical parameters and the dependence on them is complicated. A simpler example in which these ideas can be tested will be considered in a forthcoming publication.

\section{Discussion}

In this paper we have derived the first law of black hole mechanics in the context of the effective action of the Heterotic Superstring compactified on a torus at leading order in $\alpha^{\prime}$. The first law includes the variations of the conserved charges of the 1 -forms, $\mathcal{Q}_{I}$, and of the charges associated to the KR field, $\mathcal{Q}_{i}$, multiplied by the potentials $\Phi^{I}$ and $\Phi^{i}$ which are constants that we have computed on the bifurcation surface. ${ }^{16}$

The main ingredients in this proof are the identification of the parameters of the gauge transformations that generate symmetries of the complete field configurations, the careful definitions of the associated charges and the corresponding potentials through what we have called restricted generalized zeroth laws. Due to the interactions between 1-forms and the KR 2-form induced by the Chern-Simons terms, all the terms involving charges and potentials in the first law are interrelated and all their definitions are either right or wrong simultaneously. This can be seen as a test of our definitions and of the final result.

In the theory considered in this paper we have arrived at the well-known result that the entropy is one quarter of the area. In theories of higher order in the curvature it is known that there are additional contributions from the terms that contain the curvature, as the Iyer-Wald prescription makes manifest. However, as explained in the introduction, in the case of the Heterotic Superstring effective action at first order in $\alpha^{\prime}$, we also expect that the need to have well-defined charges and, simultaneously, closed forms over the bifurcation sphere will result in the need to include additional terms in the "gravitational charge" that, in the end, will give us the entropy. Work in this direction is well under way [41].

\footnotetext{
${ }^{16} \mathrm{It}$ is not hard to prove that the potentials $\Phi^{I}$, defined as the momentum maps $\mathcal{P}_{k}{ }^{I}$ are constant over the complete event horizon using the dominant energy condition and the Einstein equations as it is done for a single 1-form field in ref. [50]. It is not clear, though, how definition of the potentials $\Phi^{i}$ may be extended using other sections of the event horizon different from the bifurcation sphere because the closedness of $P_{k}+\mathcal{P}_{k I} \mathcal{F}^{I}$ is based on the property $\imath_{k} H \stackrel{\mathcal{B H}}{=} 0$. It is not clear how to extend this property to other sections of the event horizon different from the bifurcation surface $\mathcal{B H}$.
} 
Finally, we would like to comment upon two shortcomings of Wald's formalism, which, perhaps, in the end, may turn out to be only apparent: it is not clear how to include the variation of the scalar charges and the moduli $[51,52]$ in the first law. In 5 dimensions, for instance, the KR field is dual to a 1-form and black-hole solutions electrically charged with respect to this dual 1-form exist. If we describe the theory in terms of the KR 2form, it is not clear how to make the variation of this electric charge appear in the first law following this procedure. In this particular case, the electric charge of the 1-forms would be associated to S5-branes wrapped on $\mathrm{T}^{5}$ and it would be very interesting to see the precise definition of this kind of charge to try to solve the ambiguities detected in ref. [45].

\section{Acknowledgments}

TO would like to thank G. Barnich, P. Cano, P. Meessen, P.F. Ramírez, A. Ruipérez and C. Shahbazi for many useful conversations and their long-term collaboration in this research topic. DP would also like to thank G. Barnich for many useful conversations. This work has been supported in part by the MCIU, AEI, FEDER (UE) grant PGC2018-095205B-I00 and by the Spanish Research Agency (Agencia Estatal de Investigación) through the grant IFT Centro de Excelencia Severo Ochoa SEV-2016-0597. The work of ZE has also received funding from "la Caixa" Foundation (ID 100010434), under the agreement $\mathrm{LCF} / \mathrm{BQ} / \mathrm{DI} 18 / 11660042$. The work of DP is supported by a "Campus de Excelencia Internacional UAM/CSIC" FPI pre-doctoral grant. TO wishes to thank M.M. Fernández for her permanent support.

\section{A A truncation of the $d=5$ theory to a $\mathcal{N}=1, d=5$ supergravity}

A very useful, almost algorithmic, procedure has been developed in refs. [53-57] to construct supersymmetric solutions (black holes and black rings, in particular) of $\mathcal{N}=1, d=5$ supergravity coupled to vector supermultiplets. ${ }^{17}$ We can use this procedure in the context of the Heterotic Superstring Effective action compactified on a $\mathrm{T}^{5}$ if we find a consistent truncation that produces a model $\mathcal{N}=1, d=5$ supergravity. A very simple truncation with this property has been used, for instance, in ref. [58]. It can be described more conveniently as a trivial dimensional reduction on a $\mathrm{T}^{4}$ (with all the fields that arise in the reduction set to their vacuum values) followed by a non-trivial compactification on a circle. The only fields that survive are the KR 2-form (which can be dualized into a vector field), the KK and winding vectors and the dilaton and KK scalars. This field content fits into $\mathcal{N}=1, d=5$ supergravity (metric and graviphoton vector field) coupled to two vector multiplets (one vector and one real scalar field each).

In order to profit from the solution-generating techniques developed for $\mathcal{N}=1, d=5$ supergravity theories, we need to rewrite this truncated version of the Heterotic Superstring effective action in the appropriate form: first, we rewrite the action in the Einstein frame

\footnotetext{
${ }^{17}$ These are supergravities invariant under 8 independent supersymmetry transformations, which are combined in a minimal 5-dimensional spinor. Often, they are referred to as $\mathcal{N}=2, d=5$ supergravities.
} 
and then we will dualize the KR field into a vector. After that, we will identify the scalar manifold etc.

The action of the truncated theory is

$$
\begin{aligned}
S\left[e^{a}, B, \phi, k, A, B\right]= & \frac{g_{s}^{(5) 2}}{16 \pi G_{N}^{(5)}} \int e^{-2 \phi}\left[\star\left(e^{a} \wedge e^{b}\right) \wedge R_{a b}-4 d \phi \wedge \star d \phi\right. \\
& \left.+\frac{1}{2} k^{-2} d k \wedge \star d k-\frac{1}{2} k^{2} F \wedge \star F-\frac{1}{2} k^{-2} G \wedge \star G+\frac{1}{2} H \wedge \star H\right],
\end{aligned}
$$

where $H$ is simply

$$
H=d B-\frac{1}{2} A \wedge G-\frac{1}{2} B \wedge F .
$$

The string-frame Vielbein $e^{a}$ is related to the (modified) Einstein-frame Vielbein $\tilde{e}^{a}$ by

$$
e^{a}=e^{2\left(\phi-\phi_{\infty}\right) / 3} \tilde{e}^{a}, \quad g_{s}=e^{\phi_{\infty}},
$$

and the action in the (modified) Einstein frame takes the form (removing the tildes for simplicity)

$$
\begin{aligned}
S\left[e^{a}, B, \phi, k, A, B\right]= & \frac{1}{16 \pi G_{N}^{(5)}} \int\left[\star\left(e^{a} \wedge e^{b}\right) \wedge R_{a b}+\frac{4}{3} d \phi \wedge \star d \phi+\frac{1}{2} k^{-2} d k \wedge \star d k\right. \\
& \left.-\frac{1}{2} k^{2} e^{-4 \phi / 3} F \wedge \star F-\frac{1}{2} k^{-2} e^{-4 \phi / 3} G \wedge \star G+\frac{1}{2} e^{-8 \phi / 3} H \wedge \star H\right] .
\end{aligned}
$$

The next step is the dualization of the KR 2-form. As usual, we consider the above action as a functional of the 3 -form field strength $H$ and add a Lagrange-multiplier term to enforce its Bianchi identity $d H=-\frac{1}{2} \mathcal{F}_{I} \wedge \mathcal{F}^{I}$

$$
\begin{aligned}
S\left[e^{a}, H, \phi, k, A, B\right]= & \frac{1}{16 \pi G_{N}^{(5)}} \int\left[\star\left(e^{a} \wedge e^{b}\right) \wedge R_{a b}+\frac{4}{3} d \phi \wedge \star d \phi+\frac{1}{2} k^{-2} d k \wedge \star d k\right. \\
& -\frac{1}{2} k^{2} e^{-4 \phi / 3} F \wedge \star F-\frac{1}{2} k^{-2} e^{-4 \phi / 3} G \wedge \star G+\frac{1}{2} e^{-8 \phi / 3} H \wedge \star H \\
& -C \wedge(d H+F \wedge G)],
\end{aligned}
$$

where $C$ is the 1 -form dual to the 2 -form $B$. Varying this action with respect to $H$, we get

$$
\frac{\delta S}{\delta H}=e^{-8 \phi / 3} \star H-d C=0,
$$

which is solved by

$$
H=e^{8 \phi / 3} \star K, \quad K \equiv d C .
$$

Substituting this solution into the action eq. (A.5) we find the dual action

$$
\begin{aligned}
S\left[e^{a}, \phi, k, A, B, C\right]= & \frac{1}{16 \pi G_{N}^{(5)}} \int\left[\star\left(e^{a} \wedge e^{b}\right) \wedge R_{a b}+\frac{4}{3} d \phi \wedge \star d \phi+\frac{1}{2} k^{-2} d k \wedge \star d k\right. \\
& -\frac{1}{2} k^{2} e^{-4 \phi / 3} F \wedge \star F-\frac{1}{2} k^{-2} e^{-4 \phi / 3} G \wedge \star G-\frac{1}{2} e^{8 \phi / 3} K \wedge \star K \\
& -F \wedge G \wedge C] .
\end{aligned}
$$


The final step consists in finding the relation between the fields of this action and those of a $\mathcal{N}=1, d=5$ theory with two vector supermultiplets written in the standard form ${ }^{18}$

$$
\begin{aligned}
S\left[e^{a}, \phi^{x}, A^{I}\right]= & \frac{1}{16 \pi G_{N}^{(5)}} \int\left[\star\left(e^{a} \wedge e^{b}\right) \wedge R_{a b}+\frac{1}{2} g_{x y} d \phi^{x} \wedge \star d \phi^{y}-\frac{1}{2} a_{I J} F^{I} \wedge \star F^{J}\right. \\
& \left.+\frac{1}{3^{3 / 2}} C_{I J K} F^{I} \wedge F^{J} \wedge A^{K}\right],
\end{aligned}
$$

where the indices $I, J, \ldots=0,1,2$ and the indices $x, y, \ldots=1,2$. The metrics $g_{x y}(\phi), a_{I J}(\phi)$ are defined in terms of the symmetric, constant tensor $C_{I J K}$ which fully characterizes the theory and the real special geometry of the scalar manifold as follows: we start by defining 3 combinations of the 2 scalars $h^{I}(\phi)$ that satisfy the constraint

$$
C_{I J K} h^{I}(\phi) h^{J}(\phi) h^{K}(\phi)=1 .
$$

Next, we define

$$
h_{I} \equiv C_{I J K} h^{J} h^{K}, \quad \Rightarrow \quad h^{I} h_{I}=1,
$$

and

$$
h_{x}^{I} \equiv-\sqrt{3} h^{I}{ }_{, x} \equiv-\sqrt{3} \frac{\partial h^{I}}{\partial \phi^{x}}, \quad h_{I x} \equiv+\sqrt{3} h_{I, x}, \quad \Rightarrow \quad h_{I} h_{x}^{I}=h^{I} h_{I x}=0 .
$$

Then, $a_{I J}$ is defined implicitly by the relations

$$
h_{I}=a_{I J} h^{I}, \quad h_{I x}=a_{I J} h_{x}^{J} .
$$

It can be checked that

$$
a_{I J}=-2 C_{I J K} h^{K}+3 h_{I} h_{J} .
$$

The metric of the scalar manifold $g_{x y}(\phi)$, which we will use to raise and lower $x, y$ indices is (proportional to) the pullback of $a_{I J}$

$$
g_{x y} \equiv a_{I J} h_{x}^{I} h_{y}^{J}=-2 C_{I J K} h_{x}^{I} h_{y}^{J} h^{K} .
$$

If we make the identifications

$$
A^{0}=-\sqrt{3} C, \quad A^{1}=-\sqrt{3} A, \quad A^{2}=-\sqrt{3} B
$$

we find that

$$
C_{012}=1 / 6, \quad a_{00}=e^{8 \phi / 3} / 3, \quad a_{11}=k^{2} e^{-4 \phi / 3} / 3, \quad a_{22}=k^{-2} e^{-4 \phi / 3} / 3 .
$$

Since, for this $C_{I J K}$, the only non-vanishing components of $a_{I J}$ are the diagonal ones with $a_{I I}=3\left(h_{I}\right)^{2}$ we find that

$$
h_{0}=e^{4 \phi / 3} / 3, \quad h_{1}=k e^{-2 \phi / 3} / 3, \quad h_{2}=k^{-1} e^{-2 \phi / 3} / 3,
$$

\footnotetext{
${ }^{18}$ Here we are using the notation and conventions of ref. [59] with minor changes explained in appendix A of ref. [60]. See also ref. [10].
} 
which, in its turn, implies that

$$
h^{0}=e^{-4 \phi / 3}, \quad h^{1}=k^{-1} e^{2 \phi / 3}, \quad h^{2}=k e^{2 \phi / 3} .
$$

Finally, the non-vanishing components of the scalar metric are

$$
g_{\phi \phi}=8 / 3, \quad g_{k k}=k^{-2} .
$$

The equations of motion of a general $\mathcal{N}=1, d=5$ theory are (up to a global factor of $\left(16 \pi G_{N}^{(5)}\right)^{-1}$ that we omit for simplicity)

$$
\begin{aligned}
\mathbf{E}_{a}= & \imath_{a} \star\left(e^{c} \wedge e^{d}\right) \wedge R_{c d}-\frac{1}{2} g_{x y}\left(\imath_{a} d \phi^{x} \star d \phi^{y}+d \phi^{x} \wedge \imath_{a} \star d \phi^{y}\right) \\
& +\frac{1}{2} a_{I J}\left(\imath_{a} F^{I} \wedge \star F^{J}-F^{I} \wedge \imath_{a} \star F^{J}\right), \\
\mathbf{E}_{x}= & -g_{x y}\left\{d \star d \phi^{y}+\Gamma_{z w} y d \phi^{z} \wedge \star d \phi^{w}+\frac{1}{2} \partial^{y} a_{I J} F^{I} \wedge \star F^{J}\right\}, \\
\mathbf{E}_{I}= & -d\left(a_{I J} \star F^{J}\right)+\frac{1}{\sqrt{3}} C_{I J K} F^{J} \wedge F^{K} .
\end{aligned}
$$

In this action, $\phi$ stands, actually, for $\phi-\phi_{\infty}$. In other words: the field $\phi$ is constrained to vanish at infinity.

For the particular model that we have obtained as a truncation of the compactified Heterotic Superstring effective action in $d=5$ dimensions, these equations take the particular form

$$
\begin{aligned}
\mathbf{E}_{a}= & \imath_{a} \star\left(e^{c} \wedge e^{d}\right) \wedge R_{c d}-\frac{4}{3}\left(\imath_{a} d \phi \star d \phi+d \phi \wedge \imath_{a} \star d \phi\right) \\
& -\frac{1}{2} k^{-2}\left(\imath_{a} d k \star d k+d k \wedge \imath_{a} \star d k\right)+\frac{1}{6} e^{8 \phi / 3}\left(\imath_{a} F^{0} \wedge \star F^{0}-F^{0} \wedge \imath_{a} \star F^{0}\right) \\
& +\frac{1}{6} e^{-4 \phi / 3} k^{2}\left(\imath_{a} F^{1} \wedge \star F^{1}-F^{1} \wedge \imath_{a} \star F^{1}\right)+\frac{1}{6} e^{-4 \phi / 3} k^{-2}\left(\imath_{a} F^{2} \wedge \star F^{2}-F^{2} \wedge \imath_{a} \star F^{2}\right), \\
\mathbf{E}_{\phi}= & -\frac{8}{3}\left\{d \star d \phi+\frac{1}{6} e^{8 \phi / 3} F^{0} \wedge \star F^{0}-\frac{1}{12} e^{-4 \phi / 3} k^{2} F^{1} \wedge \star F^{1}-\frac{1}{12} e^{-4 \phi / 3} k^{-2} F^{2} \wedge \star F^{2}\right\} \\
\mathbf{E}_{k}= & -k^{-2}\left\{d \star d k-k^{-1} d k \wedge \star k+e^{-4 \phi / 3} k^{3} F^{1} \wedge \star F^{1}-k^{-1} e^{-4 \phi / 3} F^{2} \wedge \star F^{2}\right\} \\
\mathbf{E}_{0}= & -\frac{1}{3} d\left(e^{8 \phi / 3} \star F^{0}\right)+\frac{1}{3^{3 / 2}} F^{1} \wedge F^{2}, \\
\mathbf{E}_{1}= & -\frac{1}{3} d\left(e^{-4 \phi / 3} k^{2} \star F^{1}\right)+\frac{1}{3^{3 / 2}} F^{0} \wedge F^{2}, \\
\mathbf{E}_{2}= & -\frac{1}{3} d\left(e^{-4 \phi / 3} k^{-2} \star F^{2}\right)+\frac{1}{3^{3 / 2}} F^{0} \wedge F^{1} .
\end{aligned}
$$




\section{A.1 Further truncation to pure $\mathcal{N}=1, d=5$ supergravity}

We can truncate this theory further, to minimal (pure) supergravity as follows: if the two scalars are constant, taking into account that for $\phi$ this constant value must be $\phi=0$, (we call $k_{\infty}$ the constant value of $k$ ) their equations become the constraints

$$
\begin{aligned}
& 0=F^{0} \wedge \star F^{0}-\frac{1}{2} k_{\infty}^{2} F^{1} \wedge \star F^{1}-\frac{1}{2} k_{\infty}^{-2} F^{2} \wedge \star F^{2}, \\
& 0=k_{\infty}^{3} F^{1} \wedge \star F^{1}-k_{\infty}^{-1} F^{2} \wedge \star F^{2}
\end{aligned}
$$

whose simplest solution is this relation between vector field strengths

$$
F^{0}=k_{\infty} F^{1}=k_{\infty}^{-1} F^{2} \equiv F .
$$

Substituting this solution into the Einstein and vector equations we get only these two independent equations

$$
\begin{aligned}
\mathbf{E}_{a} & =\imath_{a} \star\left(e^{c} \wedge e^{d}\right) \wedge R_{c d}+\frac{1}{2}\left(\imath_{a} F \wedge \star F-F \wedge \imath_{a} \star F\right) \\
-\frac{2}{3} \mathbf{E} & =-\frac{1}{3} d \star F+\frac{1}{3^{3 / 2}} F \wedge F
\end{aligned}
$$

which follow from the action of minimal $d=5$ supergravity [22]

$$
S\left[e^{a}, A\right]=\frac{1}{16 \pi G_{N}^{(5)}} \int\left[\star\left(e^{a} \wedge e^{b}\right) \wedge R_{a b}-\frac{1}{2} F \wedge \star F+\frac{1}{6 \sqrt{3}} F \wedge F \wedge A\right] .
$$

The truncation procedure we have followed to arrive to this action starting from the 10dimensional Heterotic Superstring effective action can be easily reversed to embed solutions of pure $\mathcal{N}=1, d=5$ supergravity into the 10-dimensional Heterotic Superstring effective theory. In particular, we apply this recipe to the charged, non-extremal, black ring solution of ref. [46] in section 6 .

Open Access. This article is distributed under the terms of the Creative Commons Attribution License (CC-BY 4.0), which permits any use, distribution and reproduction in any medium, provided the original author(s) and source are credited.

\section{References}

[1] R.M. Wald, Black hole entropy is the Noether charge, Phys. Rev. D 48 (1993) R3427 [gr-qc/9307038] [INSPIRE].

[2] J.M. Bardeen, B. Carter and S.W. Hawking, The Four laws of black hole mechanics, Commun. Math. Phys. 31 (1973) 161 [inSPIRE].

[3] V. Iyer and R.M. Wald, Some properties of Noether charge and a proposal for dynamical black hole entropy, Phys. Rev. D 50 (1994) 846 [gr-qc/9403028] [InSPIRE].

[4] T. Jacobson and A. Mohd, Black hole entropy and Lorentz-diffeomorphism Noether charge, Phys. Rev. D 92 (2015) 124010 [arXiv:1507.01054] [INSPIRE]. 
[5] G. Barnich and G. Compere, Conserved charges and thermodynamics of the spinning Godel black hole, Phys. Rev. Lett. 95 (2005) 031302 [hep-th/0501102] [INSPIRE].

[6] G. Compere, Note on the First Law with p-form potentials, Phys. Rev. D 75 (2007) 124020 [hep-th/0703004] [INSPIRE].

[7] E. Frodden and D. Hidalgo, Surface Charges for Gravity and Electromagnetism in the First Order Formalism, Class. Quant. Grav. 35 (2018) 035002 [arXiv:1703.10120] [INSPIRE].

[8] E. Frodden and D. Hidalgo, Surface Charges Toolkit for Gravity, Int. J. Mod. Phys. D 29 (2020) 2050040 [arXiv: 1911.07264] [InSPIRE].

[9] T. Ortín, A Note on Lie-Lorentz derivatives, Class. Quant. Grav. 19 (2002) L143 [hep-th/0206159] [INSPIRE].

[10] T. Ortín, Gravity and Strings, Cambridge Monographs on Mathematical Physics, Cambridge University Press, 2nd ed. (2015) [DOI] [INSPIRE].

[11] L. Fatibene and M. Francaviglia, General theory of Lie derivatives for Lorentz tensors, Commun. Math. 19 (2011) 11 [arXiv:0904.0258].

[12] A. Lichnerowicz, Spineurs harmoniques, C. R. Acad. Sci. Paris 257 (1963) 7.

[13] Y. Kosmann, Dérivées de Lie des spineurs, C. R. Acad. Sci. Paris Sér. A 262 (1966) A289.

[14] Y. Kosmann, Dérivées de Lie des spineurs, Annali Mat. Pura Appl. (IV) 91 (1972) 317.

[15] D.J. Hurley and M.A. Vandyck, On the concepts of Lie and covariant derivatives of spinors. Part 1, J. Phys. A 27 (1994) 4569 [inSPIRE].

[16] M.A.J. Vandyck, On the problem of space-time symmetries in the theory of supergravity, Gen. Rel. Grav. 20 (1988) 261 [inSPIRE].

[17] M.A. Vandyck, On the problem of space-time symmetries in the theory of supergravity. 2: $N=2$ supergravity and spinorial Lie derivatives, Gen. Rel. Grav. 20 (1988) 905 [InSPIRE].

[18] J.M. Figueroa-O'Farrill, On the supersymmetries of Anti-de Sitter vacua, Class. Quant. Grav. 16 (1999) 2043 [hep-th/9902066] [INSPIRE].

[19] P.B. Aneesh, S. Chakraborty, S.J. Hoque and A. Virmani, First law of black hole mechanics with fermions, Class. Quant. Grav. 37 (2020) 205014 [arXiv: 2004.10215] [INSPIRE].

[20] K. Prabhu, The First Law of Black Hole Mechanics for Fields with Internal Gauge Freedom, Class. Quant. Grav. 34 (2017) 035011 [arXiv:1511.00388] [INSPIRE].

[21] S. Gao, The First law of black hole mechanics in Einstein-Maxwell and Einstein-Yang-Mills theories, Phys. Rev. D 68 (2003) 044016 [gr-qc/0304094] [inSPIRE].

[22] A. Salam and E. Sezgin, eds., Supergravities in Diverse Dimensions: Commentary and Reprints (In 2 Volumes), World Scientific, Singapore (1989) [DOI] [INSPIRE].

[23] Y. Tachikawa, Black hole entropy in the presence of Chern-Simons terms, Class. Quant. Grav. 24 (2007) 737 [hep-th/0611141] [INSPIRE].

[24] Z. Elgood and T. Ortín, T duality and Wald entropy formula in the Heterotic Superstring effective action at first-order in $\alpha^{\prime}$, JHEP 10 (2020) 097 [Erratum ibid. 06 (2021) 105] [arXiv: 2005.11272] [INSPIRE].

[25] T. Ortín, $O(n, n)$ invariance and Wald entropy formula in the Heterotic Superstring effective action at first order in $\alpha^{\prime}$, JHEP 01 (2021) 187 [arXiv: 2005.14618] [INSPIRE]. 
[26] T. Azeyanagi, R. Loganayagam, G.S. Ng and M.J. Rodriguez, Covariant Noether Charge for Higher Dimensional Chern-Simons Terms, JHEP 05 (2015) 041 [arXiv:1407.6364] [INSPIRE].

[27] Z. Elgood, P. Meessen and T. Ortín, The first law of black hole mechanics in the Einstein-Maxwell theory revisited, JHEP 09 (2020) 026 [arXiv:2006.02792] [INSPIRE].

[28] I.A. Bandos and T. Ortín, On the dualization of scalars into (d-2)-forms in supergravity. Momentum maps, R-symmetry and gauged supergravity, JHEP 08 (2016) 135 [arXiv: 1605.05559] [INSPIRE].

[29] E. Bergshoeff and M. de Roo, Supersymmetric Chern-Simons Terms in Ten-dimensions, Phys. Lett. B 218 (1989) 210 [InSPIRE].

[30] E.A. Bergshoeff and M. de Roo, The Quartic Effective Action of the Heterotic String and Supersymmetry, Nucl. Phys. B 328 (1989) 439 [inSPIRE].

[31] G. Barnich and F. Brandt, Covariant theory of asymptotic symmetries, conservation laws and central charges, Nucl. Phys. B 633 (2002) 3 [hep-th/0111246] [inSPIRE].

[32] K. Copsey and G.T. Horowitz, The Role of dipole charges in black hole thermodynamics, Phys. Rev. D 73 (2006) 024015 [hep-th/0505278] [INSPIRE].

[33] T. Regge and C. Teitelboim, Role of Surface Integrals in the Hamiltonian Formulation of General Relativity, Annals Phys. 88 (1974) 286 [INSPIRE].

[34] L.F. Abbott and S. Deser, Stability of Gravity with a Cosmological Constant, Nucl. Phys. B 195 (1982) 76 [INSPIRE].

[35] G. Barnich, Boundary charges in gauge theories: Using Stokes theorem in the bulk, Class. Quant. Grav. 20 (2003) 3685 [hep-th/0301039] [INSPIRE].

[36] D. Marolf, Chern-Simons terms and the three notions of charge, contribution to The International Conference on Quantization, Gauge Theory, and Strings: Conference Dedicated to the Memory of Professor Efim Fradkin [hep-th/0006117].

[37] R. Emparan, Rotating circular strings, and infinite nonuniqueness of black rings, JHEP 03 (2004) 064 [hep-th/0402149] [INSPIRE].

[38] D. Astefanesei and E. Radu, Quasilocal formalism and black ring thermodynamics, Phys. Rev. D 73 (2006) 044014 [hep-th/0509144] [INSPIRE].

[39] M. Rogatko, Black rings and the physical process version of the first law of thermodynamics, Phys. Rev. D 72 (2005) 074008 [Erratum ibid. 72 (2005) 089901] [hep-th/0509150] [INSPIRE].

[40] M. Rogatko, First law of black rings thermodynamics in higher dimensional dilaton gravity with $p+1$ strength forms, Phys. Rev. D 73 (2006) 024022 [hep-th/0601055] [INSPIRE].

[41] Z. Elgood, T. Ortín and D. Pereñíguez, The first law and Wald entropy formula of heterotic stringy black holes at first order in $\alpha^{\prime}$, JHEP 05 (2021) 110 [arXiv:2012.14892] [INSPIRE].

[42] P.A. Cano, S. Chimento, R. Linares, T. Ortín and P.F. Ramírez, $\alpha^{\prime}$ corrections of Reissner-Nordström black holes, JHEP 02 (2020) 031 [arXiv:1910.14324] [INSPIRE].

[43] R.R. Khuri and T. Ortín, A Nonsupersymmetric dyonic extreme Reissner-Nordstrom black hole, Phys. Lett. B 373 (1996) 56 [hep-th/9512178] [INSPIRE].

[44] A. Strominger and C. Vafa, Microscopic origin of the Bekenstein-Hawking entropy, Phys. Lett. B 379 (1996) 99 [hep-th/9601029] [INSPIRE]. 
[45] F. Faedo and P.F. Ramirez, Exact charges from heterotic black holes, JHEP 10 (2019) 033 [arXiv: 1906.12287] [INSPIRE].

[46] H. Elvang, R. Emparan and P. Figueras, Non-supersymmetric black rings as thermally excited supertubes, JHEP 02 (2005) 031 [hep-th/0412130] [INSPIRE].

[47] I. Racz and R.M. Wald, Global extensions of space-times describing asymptotic final states of black holes, Class. Quant. Grav. 13 (1996) 539 [gr-qc/9507055] [INSPIRE].

[48] J. Lee and R.M. Wald, Local symmetries and constraints, J. Math. Phys. 31 (1990) 725 [INSPIRE].

[49] J.M. Maldacena, Black holes in string theory, Ph.D. Thesis, Princeton University (1996) [hep-th/9607235] [INSPIRE].

[50] V.P. Frolov and I.D. Novikov eds., Black hole physics: Basic concepts and new developments, Fundam. Theor. Phys. 96 (1998) 1 [inSPIRE].

[51] G.W. Gibbons, R. Kallosh and B. Kol, Moduli, scalar charges, and the first law of black hole thermodynamics, Phys. Rev. Lett. 77 (1996) 4992 [hep-th/9607108] [INSPIRE].

[52] D. Astefanesei, R. Ballesteros, D. Choque and R. Rojas, Scalar charges and the first law of black hole thermodynamics, Phys. Lett. B 782 (2018) 47 [arXiv: 1803.11317] [INSPIRE].

[53] J.P. Gauntlett, J.B. Gutowski, C.M. Hull, S. Pakis and H.S. Reall, All supersymmetric solutions of minimal supergravity in five- dimensions, Class. Quant. Grav. 20 (2003) 4587 [hep-th/0209114] [INSPIRE].

[54] J.P. Gauntlett and J.B. Gutowski, General concentric black rings, Phys. Rev. D 71 (2005) 045002 [hep-th/0408122] [INSPIRE].

[55] J.B. Gutowski and W. Sabra, General supersymmetric solutions of five-dimensional supergravity, JHEP 10 (2005) 039 [hep-th/0505185] [INSPIRE].

[56] J. Bellorín and T. Ortín, Characterization of all the supersymmetric solutions of gauged $N=1, d=5$ supergravity, JHEP 08 (2007) 096 [arXiv:0705.2567] [INSPIRE].

[57] J. Bellorín, Supersymmetric solutions of gauged five-dimensional supergravity with general matter couplings, Class. Quant. Grav. 26 (2009) 195012 [arXiv:0810.0527] [INSPIRE].

[58] P.A. Cano, P. Meessen, T. Ortín and P.F. Ramírez, $\alpha^{\prime}$-corrected black holes in String Theory, JHEP 05 (2018) 110 [arXiv: 1803.01919] [INSPIRE].

[59] E. Bergshoeff, S. Cucu, T. de Wit, J. Gheerardyn, S. Vandoren and A. Van Proeyen, $N=2$ supergravity in five-dimensions revisited, Class. Quant. Grav. 21 (2004) 3015 [hep-th/0403045] [INSPIRE].

[60] J. Bellorín, P. Meessen and T. Ortín, All the supersymmetric solutions of $N=1, d=5$ ungauged supergravity, JHEP 01 (2007) 020 [hep-th/0610196] [INSPIRE]. 